\title{
Kinetic Monte Carlo modelling of dissolution and passive film formation in Fe-Cr alloys.
}

by

Amanda Jeanne Parker

\author{
A thesis \\ submitted to Victoria University of Wellington \\ in fulfilment of the \\ requirements for the degree of \\ Master of Science \\ in Physics.
}

Victoria University of Wellington

2011 


\begin{abstract}
Stainless steels differ from iron in that chromium content allows for the formation of a passive iron-chromium oxide film which is only nanometres in thickness, offering protection from the environment. While the composition of this oxide layer has been established, the mechanism of its formation is not well understood. In particular, the threshold level of chromium for oxide formation is significantly lower then the chromium content of the alloy itself. We present a Cahn Hilliard type analytical model that relates the onset of passivation to an instability which leads to a phase segregating current above $17 \% \mathrm{Cr}$ in a bulk alloy. Proposing that this current could lead to $\mathrm{Cr}$ enrichment at a surface, we compare atomistic simulations with and without a surface driven $\mathrm{Cr}$ current. We implemented a kinetic Monte Carlo algorithm with extensions to allow for vacancy assisted nearest neighbour migration in a body centered cubic alloy, tracking a surface, dissolution and surface passivation. We compare the time evolution of Fe dissolution rates, $\mathrm{Cr}$ surface enrichment and the threshold for passive film formation and find that the $\mathrm{Cr}$ current has a significant impact on each of these properties.
\end{abstract}




\section{Dedication}

I would like to dedicate this thesis to Bett and Margaret, two intelligent women who continued to learn throughout their lives.

I hope I can do so well. 


\section{Acknowledgments}

I would like to thank my supervisor, Shaun Hendy, for his invaluable advice and guidance in regard to both my project and career. I would like to gratefully acknowledge Richard Tilley and FRST for research funding. I would also like to acknowledge the applied math group at IRL, and Shaun's research group, an all round classy group of people who are always happy to help out and have hidden talents at baking. Thanks to Dmitri in particular for putting comments in his code and Nicola for making me learn latex before I realised how useful it would be. Thanks also to my friends at VUW who were around when coffee breaks were needed, and Ben for submitting this document in my absence. Lastly, thanks to my family and Tim for their help and support. 


\section{Contents}

1 Introduction $\quad 1$

2 Motivation $\quad 5$

3 Kinetic Monte Carlo methods $\quad 12$

3.1 Schulze algorithm . . . . . . . . . . . . . . . . . . . 15

3.2 Basic implementation . . . . . . . . . . . . . 16

3.2 .1 BCC Lattice . . . . . . . . . . . . . . . 16

3.2 .2 Migration rates . . . . . . . . . . . . . 18

3.2 .3 Three species . . . . . . . . . . . . . . 20

3.3 Surface implementation . . . . . . . . . . . . . . 21

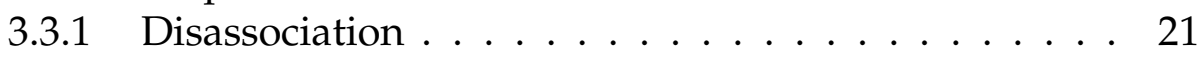

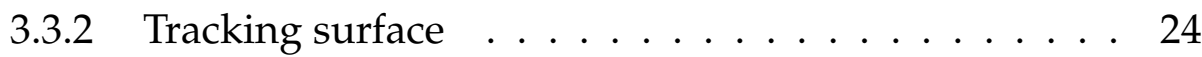

3.3 .3 Dissolution Rates . . . . . . . . . . . . . . 26

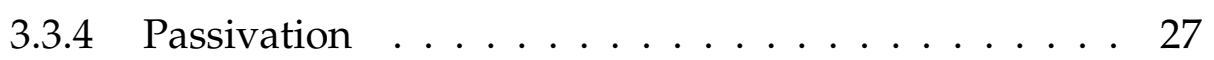

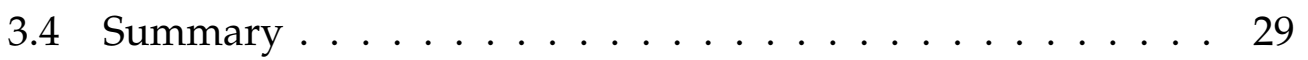

4 Results and analysis 31

4.1 Chromium current . . . . . . . . . . . . . . 31

4.1 Bulk system . . . . . . . . . . . . . . 31

4.1.2 Surface system . . . . . . . . . . . 33

4.2 Dissolution . . . . . . . . . . . . . . . . 38

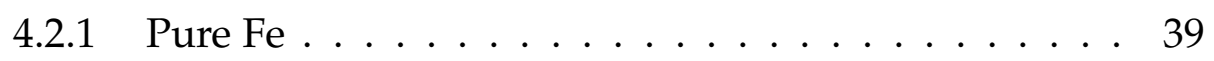

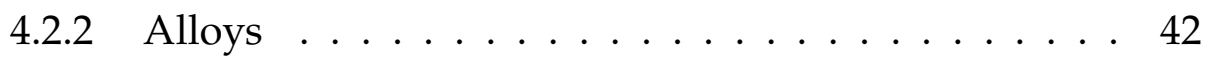

4.3 Passive film behaviour . . . . . . . . . . . . . 46

4.4 Summary ........................ 52

5 Conclusions $\quad 53$ 


\section{List of Figures}

1.1 Figure from ref. [13] The spinel crystal structure. White spheres represent tetrahedrally coordinated cations, medium size gray spheres represent octahedrally coordinated cations, larger dark spheres represent oxygen anions. . . . . . . . . 2

2.1 The excess energy of mixing $\Delta g$ for $\mathrm{Fe}_{1-\phi} \mathrm{Cr}_{\phi}$ as a function of $\phi$ (a) adapted from [7] (b) showing Redlich-Kister fit at low $\phi \ldots \ldots \ldots \ldots \ldots \ldots \ldots$

2.2 The second derivative of the excess free energy of mixing as a function of $\phi \ldots \ldots \ldots \ldots \ldots$. . . . . . . . . . . 11

3.1 (a) Shows the energy barrier, $\Delta \mathrm{E}$, crossed by an atom moving from position 1 to position 2 as shown in (b). The sites are equivalent, so the energy for relaxed state $i$ is equal to the of state $j \ldots \ldots \ldots$. . . . . . . . . . . . . . 14

3.2 A typical simulation lattice with 68921 atoms and 41 lattice planes. The lattice is (a) cubic in lattice coordinates and (b) skewed in Cartesian coordinates . . . . . . . . . . . 17

3.3 Disassociation from surface in pure Fe after $10^{6} \mathrm{KMC}$ steps

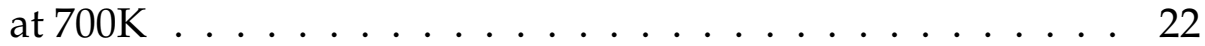

3.4 2D cubic lattice representation of disassociation limiting rules. Dashed circles are vacant sites, blue are allowed final hop sites, red are disallowed. Filled circles are atoms; blue are mobile, red are fixed. . . . . . . . . . . . . . . . . . 23

3.5 BCC cell with a $<110>$ surface plane. No nearest neighbours are perpendicular to the surface plane. . . . . . . . . . 24 
3.6 Proportion of migration events which occur with a set number of vacant nearest neighbours compared to the bulk concentration of vacancies in pure Fe at 700K. . . . . . . . . . . 25

4.1 Pure Fe with $10 \%$ of lattice sites vacant. Vacancies are shown in black (a) initial random distribution (b) final distribution of vacancies after $7 \mu$ s, aggregation of vacancies is observed.

4.2 Distribution of $\mathrm{Cr}$ in planes parallel to the surface after $10^{7}$ computational steps at temperatures (a) $700 \mathrm{~K}$ (b) $500 \mathrm{~K}$ (c) $300 \mathrm{~K}$. The legend gives the percentage of $\mathrm{Cr}$ in the alloy. $41 \times 41 \times 34$ block . . . . . . . . . . . . . . . . .

4.3 Evolution of $\% \mathrm{Cr}$ in the surface plane of an $\mathrm{Fe}_{0.85} \mathrm{Cr}_{0.15}$ alloy over time at $700 \mathrm{~K}$ when in the initial configuration (a) $10 \%$ of surface sites are vacant (b) the same number of vacancies are spread throughout the alloy . . . . . . . . . .

4.4 Total dissolved Fe in a pure Fe lattice containing 34 planes parallel to the $\langle 110\rangle$ surface, each containing 1681 atoms (a) $\nu_{1}=10^{-4}$ (b) $\nu_{1}=10^{-2} \ldots \ldots \ldots \ldots \ldots \ldots$. . . . . . . . . . .

4.5 Dissolution in pure Fe at 700K with $\nu_{1}=10^{-4}$ at (a) $t=0$, no dissolved Fe (b) $t=1.2 \mathrm{~ms}$, the number of dissolved Fe atoms is equal to half the total number in a plane (c) $t=$ $1.9 \mathrm{~ms}$, the number of dissolved Fe atoms is equal to the total number in a plane. The block has 34 planes parallel to the $<110>$ surface, each containing 1681 atoms . . . . . . . . .

4.6 Total Fe dissolution over time in pure $\mathrm{Fe}$ and $\mathrm{Fe}_{85} \mathrm{Cr}_{15}$ with and without $\mathrm{Cr}$ current at $700 \mathrm{~K}$. The triangles show the point at which surface $\mathrm{Cr}$ content has increased from $15 \%$ to $23 \%$. The simulation block is 34 planes parallel to the $<110\rangle$ surface, each containing 1681 atoms . . . . . . . . . .

4.7 Rate of Fe dissolution at $\nu_{1}=10^{-4}$, with and without $\mathrm{Cr}$ current, only Fe dissolution, and Fe dissolution as well as $\mathrm{Cr}$ dissolution at $\nu_{1}=10^{-5}$, at $700 \mathrm{~K}$ with 34 atomic planes containing 1681 atoms. . . . . . . . . . . . . .

4.8 Surface $\mathrm{Cr}$ enrichment in $\mathrm{Fe}_{85} \mathrm{Cr}_{15}$ at varied $\nu_{1}$ with and without $\mathrm{Cr}$ current at $700 \mathrm{~K}$ in a $41 \times 41 \times$ simulation block. . . 
4.9 (a) Total Fe dissolution and (b) dissolution rate over time in alloys for bulk $\mathrm{Cr}$ content varied from $5 \%$ to $25 \%$ with the local environment threshold for passivation set at $20 \%$ $\mathrm{Cr}$, at $700 \mathrm{~K}$ with 34 planes parallel to the surface containing 1681 atoms each. A clear change in behaviour is observed between $8 \%$ to $10 \%$ bulk $\mathrm{Cr}$ content. . . . . . . . . . . . 47

$4.10700 \mathrm{~K}$, local threshold for passivation is $20 \%$ (a) $\mathrm{Fe}_{92} \mathrm{Cr}_{8}$ continued dissolution (b) $\mathrm{Fe}_{90} \mathrm{Cr}_{10}$ a passive film forms. Atoms colours; blue $=\mathrm{Fe}$, red $=\mathrm{Cr}$, green $=$ passive $\mathrm{Fe}$, yellow $=$ passive Cr. . . . . . . . . . . . . . . . . 48

4.11 Comparison of passivation in (a) mobile-Cr regime (b) Felike regime, for fixed alloy mixtures from $5 \% \mathrm{Cr}$ content to $25 \% \mathrm{Cr}$ content at $700 \mathrm{~K}$. The simulation block has 34 planes parallel to the surface containing 1681 atoms each. . . . . . . 50

\section{List of Tables}

2.1 Expansion coefficients for $\Delta g$ in equations $2.3\left\{L_{n}\right\}$, and $2.12\left\{G_{n}\right\}$ from Ref. [7] . . . . . . . . . . . . . . 6

3.1 Possible site occupancies and associated events within the KMC code, where 'type' is the numeric descriptor and ' $v$ ' is the number of vacant nearest neighbours $\ldots \ldots$. . . . . 30

4.1 Minimum bulk $\mathrm{Cr}$ content required for passive film to form when the local passivation threshold is $20 \%$. . . . . . . 51 


\section{Chapter 1}

\section{Introduction}

A wide variety of stainless steels have been developed for use in industrial and domestic applications. Stainless steels contain chromium along with some fraction of a range of other elements such as molybdenum, nickel, manganese or carbon dependant on the desired physical properties. In essence though, stainless steels differ from iron in that alloying allows for the formation of a passive oxide film which is only nanometres in thickness. This film offers protection from the environment resulting in high corrosion resistance in both humid and neutral aqueous environments [2]. It has been established experimentally that a relatively small chromium content in the alloy is required for a passive film to form, $12 \%-17 \%$ and that the transition in behavior is quite sharp [21].

Given the prevalence of stainless steels, a thorough understanding of the mechanisms and limitations of this corrosion resistance is desirable. For example, consider the stainless steels in nuclear power plants. They must be able to withstand neutron bombardment, and high temperatures. It is imperative to have a understanding of the material's lifetime in these conditions [31,9]. Unsurprisingly then, the stucture and formation of this oxide film along with the conditions under which passivity is conferred have been studied both experimentally and through first principles calculations. 


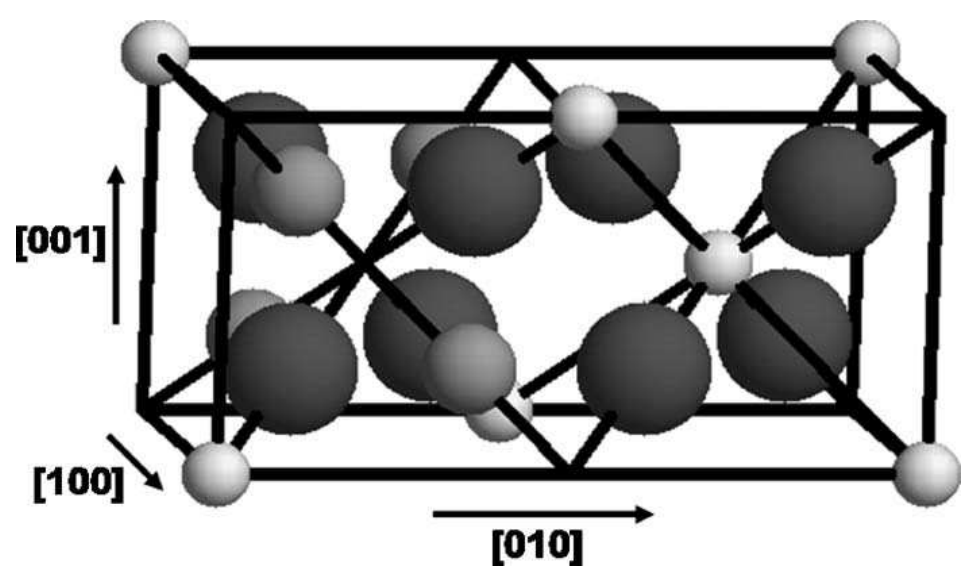

Figure 1.1: Figure from ref. [13] The spinel crystal structure. White spheres represent tetrahedrally coordinated cations, medium size gray spheres represent octahedrally coordinated cations, larger dark spheres represent oxygen anions.

Experimentally, films are typically studied in a borate buffer at varying $\mathrm{pH}$. On pure Fe the film that forms at $\mathrm{pH} 8.4$ has a structure similar to the inverse spinel structure of magnetite, $\mathrm{Fe}_{3} \mathrm{O}_{4}$ [11]. In the case of alloys, the structure has been found to fall between magnetite and spinel chromite, $\mathrm{Fe}-\mathrm{Cr}_{2} \mathrm{O}_{4}$, with varying values of $\mathrm{x}$ in composition $\mathrm{Fe}_{3-2 x} \mathrm{Cr}_{2 x} \mathrm{O}_{4}$. The difference between a spinel and an inverse spinel is not in the crystallographic structure, an image of which is shown in figure 1.1. Rather, in a spinel the trivalent cations occupy octahedral sites, and divalent cations occupy tetrahedral sites. In an inverse spinel, half the trivalent cations share the available tetrahedral sites with the divalent cations, with the remaining half occupying octahedral sites [13].

It has also been established that the $\mathrm{Cr}$ content of the oxide film is enriched compared to the alloy. Oblonsky et al. [18, 20] found passivation was accompanied by dissolution of Fe; a small amount at pH 5.5-7.5 and significantly more at lower $\mathrm{pH} 4.5$. In both cases though, the film is enriched in $\mathrm{Cr}$ compared to the content in the alloy. Davenport et al. [11] investigate passivity at a set $\mathrm{pH}$ of 4.5 and applied potential $-0.07 \mathrm{~V}$ estab- 
lishing that an alloy must contain at least $15 \% \mathrm{Cr}$ content to confer passivity in these conditions. First principles calculations replicating these conditions confirm that the minimum thermodynamically stable oxide must contain $x=0.3$, that is $23 \% \mathrm{Cr}$ content [13]. However, while the composition of this oxide layer has been established, the mechanism of its formation is not well understood, in particular it is not yet known how this surface enrichment occurs.

Regarding the kinetics of film growth, it has been found that while passive film growth occurs in seconds or minutes, long range ordering of the film takes several hours. Initially small $(3 \mathrm{~nm})$ crystalline regions form, conferring passivity, long range ordering of these regions requires the greater time scale [21].

Despite the body of work in this area, the origin of the critical Cr content required for passive film formation is not well understood. Several models have been proposed to explain the onset of passive film formation. The earliest considers the percolation threshold of $\mathrm{Cr}$ in the alloy [27, 23]. If the $\mathrm{Cr}$ concentration is sufficiently high, a Cr-Cr network forms through the alloy so that only finite clusters of Fe dissolve leaving chromium rich oxide which passivates the metal. Similarly, later work by McCafferty $[16,17]$ considers a network of $\mathrm{Cr}$ ions in the passive film itself as necessary for passivity. McCafferty does not describe the mechanism by which the $\mathrm{Cr}$ network provides passivity. Most recently Monte-Carlo computational modelling has been applied [15], however, like the earlier models the only mechanism for $\mathrm{Cr}$ surface enrichment allowed is selective Fe dissolution. This is insufficient to account for the passivity of $\mathrm{Fe}-\mathrm{Cr}$ alloys at high temperatures in dry conditions where limited or no dissolution of $\mathrm{Fe}$ takes place [19]. Additionally, some dissolution of $\mathrm{Cr}$ occurs experimentally, with up to $10 \%$ dissolved material being $\mathrm{Cr}$ [12].

This thesis relates the onset of passive film formation to an instability in the alloy itself which leads to a current of $\mathrm{Cr}$ to the surface contributing to surface $\mathrm{Cr}$ enrichment and passive film formation. 
We present an analytical continuum model for the time evolution of the distribution of $\mathrm{Cr}$ within an Fe-Cr alloy. We model the free energy of the alloy, with parameters calculated from first principles electronic structure calculations, to derive a Cahn-Hilliard model for phase separation in the alloy. We find that this equation shows an instability at $17 \% \mathrm{Cr}$ content which drives a current of $\mathrm{Cr}$. This current of $\mathrm{Cr}$ could lead to enrichment of $\mathrm{Cr}$ at the surface above this threshold level.

We then take an atomistic approach to investigating the impact of a $\mathrm{Cr}$ current on surface $\mathrm{Cr}$ enrichment and passive film formation. The methodology we use is kinetic Monte Carlo (KMC), a stochastic algorithm for modelling dynamic atomistic systems. Kinetic Monte Carlo allows for the fast simulation of events based on fixed transition rates between equilibrium states. We build a KMC model which allows for diffusion, dissolution and passivation in Fe-Cr alloys, in order to investigate the impact of a Cr current on passive film formation. Rather than considering an explicit solvent with ions dissolving into it, we treat dissolution of the solid as atoms being removed from the system.

Chapter 2 gives the details the analytical model and the derivation of the instability threshold. Chapter 3 first gives an introduction to KMC and transition state theory (TST), and then describes the details of our implementation. Chapter 4 presents and discusses results of KMC simulations and chapter 5 concludes this work. 


\section{Chapter 2}

\section{Motivation}

Previous models of the passivity of Fe-Cr alloys have been based on the idea that a static network of $\mathrm{Cr}$ conveys stability [27, 23, 16, 17]. In this section we will investigate the possibility that the migration of $\mathrm{Cr}$ can also play a role. Namely, we investigate the possibility that an instability in the alloy can deliver high concentrations of $\mathrm{Cr}$ to the alloy surface during the dissolution process. Starting from a model for the free energy of the alloy with parameters derived from first principles electronic structure calculations, we derive a differential equation that describes the time evolution of inhomogeneities in $\mathrm{Cr}$ concentration. We show this equation is unstable above $17 \%$ chromium content in the alloy and illustrate how this instability can promote the growth of a passive oxide film.

For an iron-chromium alloy, $\mathrm{Fe}_{1-\phi} \mathrm{Cr}_{\phi}$, we can express the free energy of formation, as

$$
g(\phi)=\phi g_{C r}+(1-\phi) g_{F e}+g_{m i x}+\Delta g
$$

where $\phi g_{C r}+(1-\phi) g_{F e}$ is the weighted average of the free energy of the pure components, $g_{m i x}$ is the entropic free energy term for mixing of a random alloy, and $\Delta g$ is the excess free energy of mixing. 


\begin{tabular}{c|cc}
\hline \hline & & \\
& $L_{n}$ & $G_{n}$ \\
\hline & & \\
0 & 0.415660 & -0.16130 \\
1 & 0.0814134 & 3.71417 \\
2 & -0.0101899 & -9.12960 \\
3 & 0.267659 & 10.08588 \\
4 & -0.248269 & -3.97230 \\
& & \\
\hline \hline
\end{tabular}

Table 2.1: Expansion coefficients for $\Delta g$ in equations $2.3\left\{L_{n}\right\}$, and 2.12 $\left\{G_{n}\right\}$ from Ref. [7]

$g_{m i x}$ can be described as the ideal free energy of mixing

$$
g_{m i x}=k T[\phi \ln \phi+(1-\phi) \ln (1-\phi)]
$$

which is derived by considering the number of ways of arranging the different atoms for a given value of $\phi$ [14].

Here we make two important simplifications, $g_{\text {mix }}$ is neglected given that at ambient temperatures $\Delta g \gg g_{m i x}$, and we assume that the formation energy does not depend on temperature. Given the latter, $\Delta g$ is approximately equivalent to $\Delta H$ which can be calculated by ab initio methods by taking the binding energy of the alloy less a weighted average of the binding energies for pure Fe and $\mathrm{Cr}$. A fit to such $a b$ initio data for ferromagnetic Fe-Cr alloys can be reproduced by a Redlich-Kister expansion to 4 th order [25]

$$
\Delta g=\phi(1-\phi) \sum_{i}^{N} L_{i}(2 \phi-1)^{i}
$$

We will use the coefficients $L_{i}$ proposed by Caro et al [7], shown in table 2.1. Figure 2.1 plots $\Delta g$ as a function of $\phi$, showing $a b$ initio data points from [21] and fitted with this expansion. For high $\mathrm{Cr}$ content, the anti- 
ferromagnetic alignment of $\mathrm{Cr}$ becomes dominant, and the trend of $\Delta g$ alters from this fit. We are however, interested in values of $\phi$ well within the fitted range. Interestingly, the curve is asymmetric. At low $\mathrm{Cr}$ content, $\Delta g$ becomes negative, down to $-8 \mathrm{meV} /$ atom, meaning in this region an alloy is energetically favourable as compared to it's constituent parts. At higher $\mathrm{Cr}$ concentrations the free energy does not favour dissolution of $\mathrm{Cr}$ in Fe.

We now consider an inhomogeneous alloy so let, $\phi=\phi(\mathbf{r})$. The dynamics of diffusion in the alloy are dictated by it's attempt to minimise the free energy, which now has functional form $G[\phi(\mathbf{r})]$. We also include a term to account for interfacial tension $a^{2}(\nabla \phi)^{2}$ giving

$$
G[\phi]=\int d^{3} \mathbf{r} \rho\left[g(\phi)+a^{2}(\nabla \phi)^{2}\right]
$$

where $\rho$ is the number density (which we assume remains constant) and $a$ is a length scale related to the interfacial tension.

Given that $\phi$ is a conserved physical quantity, we can formulate a CahnHilliard model, which applies to any situation with conserved diffusive dynamics in a scalar field, but was in fact derived to model phase separation in binary alloys [6].

We begin with the conservation law for $\phi$,

$$
\frac{\partial \phi}{\partial t}+\nabla \cdot \mathbf{J}=0
$$

ensuring that the change in $\phi$ in any particular volume is the difference between the current $(\mathbf{J})$ flowing in and out.

Secondly, we must consider the form this current should take. It should not depend on position explicitly, and should act to minimise the free energy. So, there should be flow from areas of high to low chemical potential, 


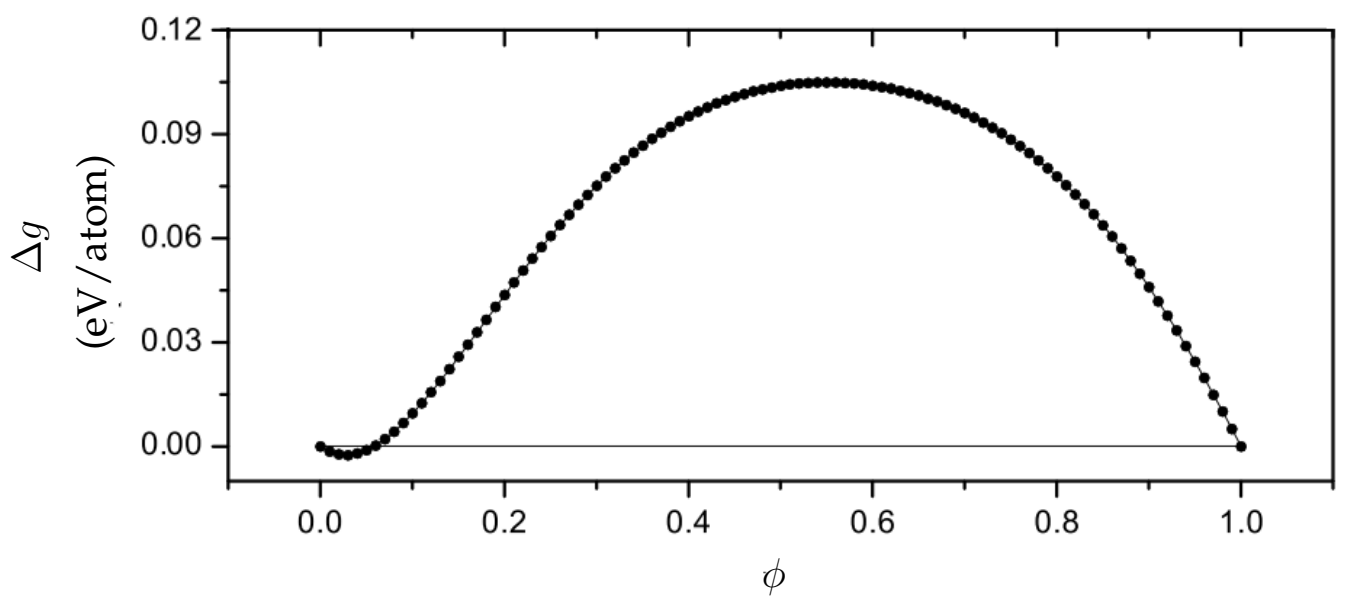

(a)

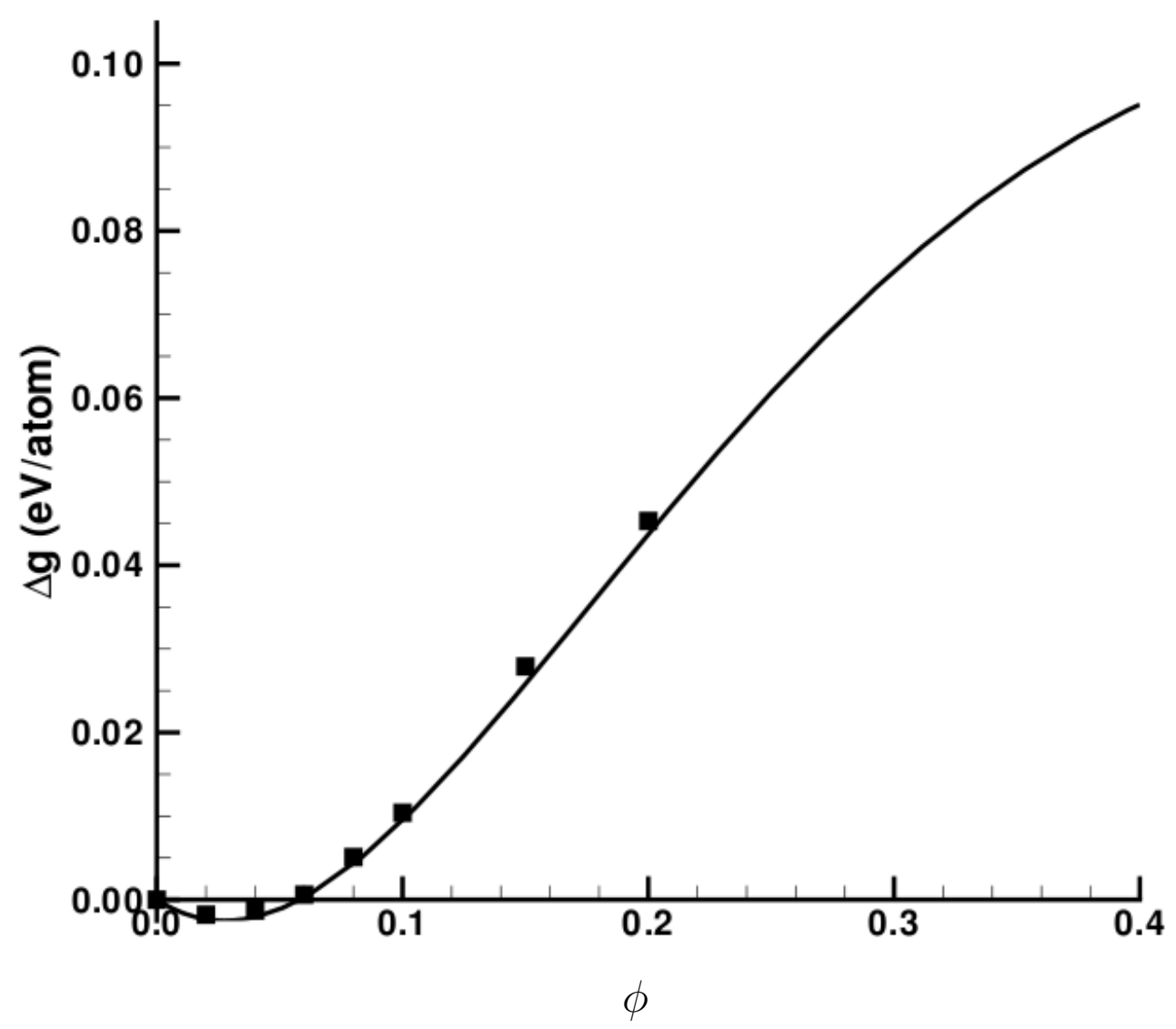

(b)

Figure 2.1: The excess energy of mixing $\Delta g$ for $\mathrm{Fe}_{1-\phi} \mathrm{Cr}_{\phi}$ as a function of $\phi$ (a) adapted from [7] (b) showing Redlich-Kister fit at low $\phi$. 
$(\mu)$

$$
\mu=\frac{\delta G[\phi]}{\delta \phi}
$$

The simplest form of $\mathbf{J}$ which suffices is

$$
\mathbf{J}=-M \nabla \mu
$$

where $M$ is the mobility, which must be a positive function of $\phi$.

Now, we can derive expressions for the dynamics of the alloy. Firstly we obtain the chemical potential

$$
\mu=\rho\left(g_{C r}-g_{F e}+\frac{d \Delta g}{d \phi}-2 a^{2} \nabla^{2} \phi\right)
$$

Substituting the chemical potential into 2.7 gives

$$
\mathbf{J}=-\rho M\left(\frac{d^{2} \Delta g}{d \phi^{2}} \nabla \phi+a^{2} \nabla^{3} \phi\right)
$$

where the current consists of a non-linear diffusion term (linear in $\nabla \phi$ ) and a surface tension term (linear in $\nabla^{3} \phi$ ).

Finally, we consider the diffusion coefficient $[D(\phi)]$ which is related to J by

$$
\mathbf{J}=-D(\phi) \nabla \phi
$$

Intuitively, a positive diffusion coefficient describes the flow from areas of high concentration to low, leading toward a stable mixture. We however, are interested in the range of $\phi$ which gives a negative diffusion coefficient, which will lead to phase separation i.e. spatial segregation of the different components of the alloy. This is consistent with our previous observation that the excess free energy does not favour the dissolution of $\mathrm{Cr}$ in Fe at high concentrations. Given we are interested in the onset of this behaviour, we can assume our initial state is homogenous, so there is no significant phase separation. Based on this assumption, we can neglect the 
$a^{2} \nabla^{3} \phi$ interfacial term from 2.9. Now comparing 2.9 to 2.10 determines the diffusion coefficient as

$$
D(\phi)=\rho M\left(\frac{d^{2} \Delta g}{d \phi^{2}}\right)
$$

So, the diffusion coefficient becomes negative if the second derivative of the free energy is negative. Referring back to figure 2.1, we are interested in the inflection point of the $\Delta g$ curve. Expanding out our expression for $\Delta g$ to be in terms of $\phi$ rather than $(2 \phi-1)$ we have

$$
\Delta g=\phi(1-\phi) \sum_{i}^{4} G_{i} \phi^{i}
$$

with new coefficients $G_{i}$ given in table 2.1. The second derivative is then

$$
\frac{d^{2} \Delta g}{d \phi^{2}}=2\left(G_{1}-G_{0}\right)+6\left(G_{2}-G_{1}\right) \phi+12\left(G_{3}-G_{2}\right) \phi^{2}+20\left(G_{4}-G_{3}\right) \phi^{3}-30 G_{4} \phi^{4}
$$

which is plotted in 2.2. $\frac{d^{2} \Delta g}{d \phi^{2}}$ is negative above $17 \% \mathrm{Cr}$ content, implying an instability in alloys of this composition, causing phase separation. This percentage is in the range of the onset of passive film formation, which suggests that if we introduce a surface the current that is caused by this instability will result in a net migration of chromium to the surface. This would allow for the build up of excess $\mathrm{Cr}$ at the surface, sufficient for the formation of a thermodynamically stable oxide layer.

An extension of the above analysis to include a surface quickly becomes difficult. However, because this system is intrinsically atomistic we are strongly motivated to try an atomistic modelling approach to investigate this instability further. Chapter 3 will present our atomistic modelling approach which will be applied to $\mathrm{Fe}-\mathrm{Cr}$ alloys in chapter 4 . 


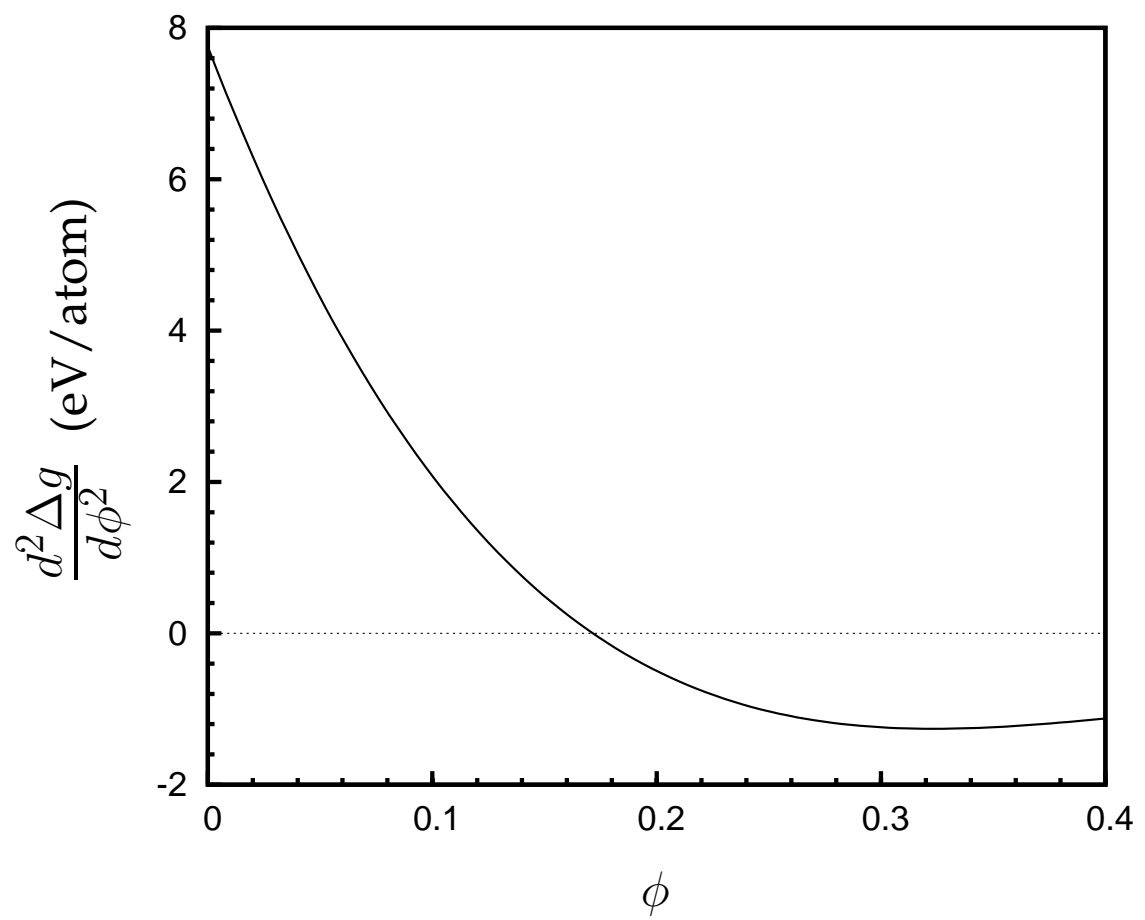

Figure 2.2: The second derivative of the excess free energy of mixing as a function of $\phi$. 


\section{Chapter 3}

\section{Kinetic Monte Carlo methods}

The methodology we used to build our computational model was kinetic Monte Carlo, a fast stochastic method for modelling dynamic atomistic systems. This chapter will first give a brief introduction to KMC and transition state theory primarily based on the approach by A. Voter [30], followed by the specifics of our Fe-Cr alloy model.

To outline the KMC method, consider a lattice of atoms in a geometry relaxed in terms of energy, state $i$. At a finite temperature, the atoms will vibrate. This does not constitute a change in state. If we were to minimise the energy the atoms would return to their initial configuration. The system as a whole has remained in the same potential energy well. Occasionally an atom might oscillate far enough so that it hops to a new lattice site. When relaxed, the system then has a different configuration $j$, having overcome an energy barrier $\Delta E$ and sits in a different potential well. In this case, we now consider that the system has changed state.

Given the difference in time scales between vibrations and state transitions, the system has no memory of how it came to be in the present state. The trajectory from state to state is therefore a random walk, and the probability of the system moving to a new state in any short increment of time is the same as the previous increment. This gives rise to exponential decay statistics (analogous to nuclear decay), relating the probability of escaping 
a state to the total rate $R$ for escape from the state. The probability that the system remains in state $i$ of the system for a time $t$

$$
p_{\text {survival }}(t)=\exp (-R t) .
$$

The total rate $R$ is the sum of the rates $R_{i j}$, where $j$ is any possible configuration the system can escape to from $i$.

It is then easy to envisage an algorithm that allows us to propagate the system from state to state in a stochastic manner, such that the probability of a particular event being executed is proportional to the corresponding rate. The so called rejection free, or Bortz, Karlos, and Lebowitz (BKL) algorithm [5], is described below

1. Calculate the total rate $R=\sum_{n=1}^{N} R_{n}$ retaining the partial sums $S_{j}=$ $\Sigma_{n=1}^{j} R_{n}$.

2. Select a random number $r \in[0,1)$

3. Search through the list of partial sums until $r R<S_{j}$

4. Execute event $j$ and update all rates appropriately

After each KMC step, time is iterated by an amount $d t$,

$$
d t=\frac{1}{R}
$$

While simplistic, this time iteration makes intuitive sense. If two events occur at differing rates, the time average of something occurring is obviously less than the period of either event.

Transition state theory allows us to calculates the rates $R_{i j}$ for each event, by considering the flux through a saddle point on the energy surface such that

$$
R_{i j}=\nu_{0} \exp \left[\frac{-\Delta E}{k_{b} T}\right]
$$


where $\nu_{0}$ is an attempt frequency on the order of atomic vibrations, usually taken as $10^{13} s^{-1}$ for diffusion events [30]. The energy barriers can be calculated outside of the KMC algorithm, and included using a rate cata$\log$, or bond counting scheme. Figure 3.1 gives an example of an energy barrier for an atom diffusing to a similar site.

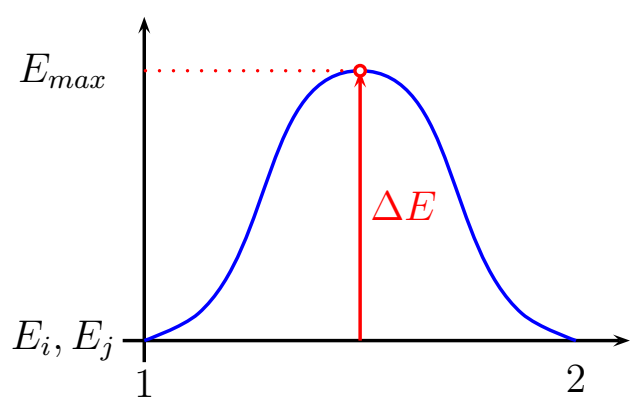

(a)

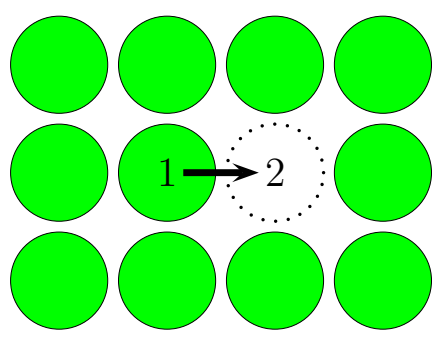

(b)

Figure 3.1: (a) Shows the energy barrier, $\Delta \mathrm{E}$, crossed by an atom moving from position 1 to position 2 as shown in (b). The sites are equivalent, so the energy for relaxed state $i$ is equal to the of state $j$.

A molecular dynamics (MD) simulation would evolve the system by solving Newton's equations of motion with an inter-atomic potential. While time steps would be limited to less than that of atomic vibrations, the full dynamics of the system can be calculated. Kinetic Monte Carlo allows us to model the infrequent events without the computational expense of solving the full equations of motion, or the restrictive time step required to resolve atomic vibrations. The penalty for the lower computational expense is a loss of generality. All possible events must be predetermined, meaning no surprising transport mechanisms will be revealed.

The starting point for our KMC model was code developed by Dmitri Scherbachov and Tim Schulze [26]. Their KMC code was designed to 
model melting of nanoclusters on a face centered cubic (FCC) lattice. It allowed for phase change and diffusion events for two species in a fixed energy regime. This section will describe the implemented KMC algorithm, extensions made to the KMC code, and the measurement of physical parameters.

\subsection{Schulze algorithm}

While the original KMC algorithm in itself has a low computational expense, it can be further improved upon. If we consider $M$ independent processes, the BKL algorithm scales as $\mathcal{O}(M)$, or, with an efficient binary search such as the method of Blue, Beichl and Sullivan [3], $\mathcal{O}(\log M)$. The Schulze algorithm [26], minimises searching by grouping similar events, those with the same rate, together in an ordered list, which is in theory independent of $M$. In practise, increased memory requirements do impose some expense that scales with $M$.

The simulations here utilised a crystal lattice which is built into the algorithm. Essentially, a fixed array of lattice sites are considered and their occupation and associated rates updated throughout the simulation. This approach is simpler, and computationally more efficient than tracking the location of particular atoms throughout the simulations.

Rates are considered to depend on the local environment of a site, which limits the number of different processes $N$, such that $N \ll M$. That is, are fewer tsypes of events $(N)$ than the total number of possible events $(M)$. An ordered list of similar lattice sites is maintained, as well as an index of the partitions from which the counts $C_{n}$ of the number of ways event $n$ could occur is obtained. The algorithm then proceeds as follows,

1. Calculate the total rate

$$
R=\Sigma_{n=1}^{N} R_{n} C_{n}
$$


retaining the partial sums $S_{n}$.

2. Select a random number $r \in[0, R)$

3. Search through the list of partial sums $S_{n}$ until $r<S_{n}$

4. Select an event $(m)$ from the set of events that occur at this rate by computing

$$
m=\operatorname{int}\left(\frac{S_{n}-r}{R_{n}}\right)+1
$$

5. Execute that event and update the local environment

6. For the events that have their rates changed from $R_{n_{i}}$ to $R_{n_{f}}$ (a) move them up/down the list to the appropriate partition and update the list index (b) increase $C_{n_{f}}$ by 1 , and decrease $C_{n_{i}}$ by 1

Note that, when an event is selected in 3 the search is through a short list. When an event is chosen from the subset in 4 it is by calculation rather than search. This is where the computational saving is made. The update in step 6 is further complicated by keeping track of a surface and passivation, this will be expanded upon in section 3.3.

\subsection{Basic implementation}

\subsubsection{BCC Lattice}

The ground state crystal structure for both pure $\mathrm{Fe}$ and $\mathrm{Cr}$ and alloys is body centered cubic (BCC), with experimentally and theoretically determined lattice parameters $(a)$ ranging from $2.84 \AA$ to $2.87 \AA$ for pure Fe through to $2.85 \AA$ to $2.88 \AA$ for pure Cr [32].

Any regular crystal lattice can be constructed by a tiling of a basis set of sites using a linear combination of primitive lattice vectors. A BCC lattice can be visualised by considering a cubic lattice with an additional atom at the centre of each cube. These central atoms form a second cubic lattice. 
However, rather than considering the BCC lattice as tiled cubically, which would require a two atom basis set, we use a single atom basis set $\mathrm{b}_{1}=\mathbf{0}$, and the lattice vectors

$$
\begin{aligned}
& \mathbf{a}_{\mathbf{1}}=\frac{a}{2}(-\hat{\mathbf{x}}+\hat{\mathbf{y}}+\hat{\mathbf{z}}) \\
& \mathbf{a}_{\mathbf{2}}=\frac{a}{2}(\hat{\mathbf{x}}-\hat{\mathbf{y}}+\hat{\mathbf{z}}) \\
& \mathbf{a}_{\mathbf{3}}=\frac{a}{2}(\hat{\mathbf{x}}+\hat{\mathbf{y}}-\hat{\mathbf{z}})
\end{aligned}
$$

where $\hat{\mathbf{x}}, \hat{\mathbf{y}}, \hat{\mathbf{z}}$ are the Cartesian unit vectors, and $a$ is the lattice parameter.

Within simulations lattice coordinates were used. However, for visualisation where a standard xyz file format is required, we converted back to Cartesian coordinates. Our simulation lattice is cubic in lattice coordinates, there are an equal number of sites each of the lattice directions. The lattice becomes skewed in Cartesian coordinates as shown in figure 3.2.

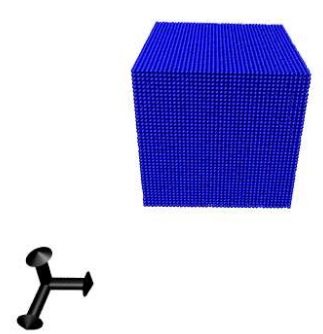

(a)

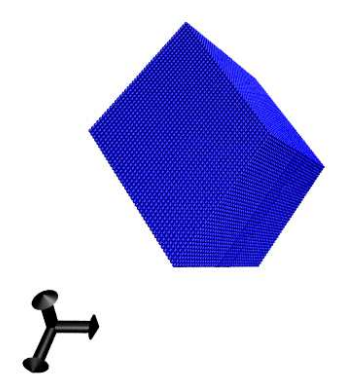

(b)

Figure 3.2: A typical simulation lattice with 68921 atoms and 41 lattice planes. The lattice is (a) cubic in lattice coordinates and (b) skewed in Cartesian coordinates

It was particularly important to consider the nearest neighbours of a particular site, given that we implemented nearest neighbour migration, 
and the nearest neighbour environment is considered in the bond counting scheme. The set of eight nearest neighbours for any given site in lattice coordinates is

$$
\left\{\mathrm{nn}_{i=1}^{8}\right\}=\left\{ \pm\left(\begin{array}{l}
1 \\
0 \\
0
\end{array}\right), \pm\left(\begin{array}{l}
0 \\
1 \\
0
\end{array}\right), \pm\left(\begin{array}{l}
0 \\
0 \\
1
\end{array}\right), \pm\left(\begin{array}{l}
1 \\
1 \\
1
\end{array}\right)\right\}
$$

We also consider the 6 second nearest neighbours,

$$
\left\{2 \mathrm{nn}_{i=1}^{6}\right\}=\left\{ \pm\left(\begin{array}{l}
1 \\
1 \\
0
\end{array}\right), \pm\left(\begin{array}{l}
1 \\
0 \\
1
\end{array}\right), \pm\left(\begin{array}{l}
0 \\
1 \\
1
\end{array}\right)\right\}
$$

and the 12 third nearest neighbours

$$
\left\{3 \mathrm{nn}_{i=1}^{12}\right\}=\left\{ \pm\left(\begin{array}{l}
2 \\
1 \\
1
\end{array}\right), \pm\left(\begin{array}{l}
1 \\
2 \\
1
\end{array}\right), \pm\left(\begin{array}{l}
1 \\
1 \\
2
\end{array}\right), \pm\left(\begin{array}{c}
1 \\
-1 \\
0
\end{array}\right), \pm\left(\begin{array}{c}
1 \\
0 \\
-1
\end{array}\right), \pm\left(\begin{array}{c}
0 \\
1 \\
-1
\end{array}\right) .\right\}
$$

\subsubsection{Migration rates}

In our simulations, a diffusion event is explicitly a solid atom moving from an initial lattice site to a vacant final site. Following the Schulze algorithm, similar sites have the same possible event rates and are grouped together in an ordered list. The number of diffusion rates is determined by the possible configurations from which a hop can occur. A diffusion event can occur if a site is occupied by $\mathrm{Fe}$ or $\mathrm{Cr}$ and has between one and eight vacant nearest nieghbours. Each non-vacant nearest nieghbour can be either Fe or $\mathrm{Cr}$, so we have $2 \times \sum_{i=1}^{8} i=72$ diffusion rates.

The values of the energy barriers, $\Delta E$, for Fe-Cr systems have, in some cases, been well established through density functional theory calculations and MD modelling. Energy barriers for vacancy assisted nearest neigh- 
bour migration for $\mathrm{Fe}$ and $\mathrm{Cr}$ in a pure Fe background are accepted to be in the range of $0.65 \mathrm{eV}$ to $0.67 \mathrm{eV}$ and $0.52 \mathrm{eV}$ to $0.56 \mathrm{eV}$, respectively $[22,4]$. Note that $\mathrm{Cr}$ is more mobile than $\mathrm{Fe}$.

However, we require variation in $\Delta E$ dependant on the concentration of $\mathrm{Cr}$ in the local environment and also expect lower energy barriers at the surface. We therefore calculate the energy barriers $\Delta E$ using a typical bond counting scheme, each nearest neighbour bond to the initial site is considered to linearly contribute to $\Delta E$ [10]. The energy barrier for a $\mathrm{Cr}$ atom to move to a vacant nearest neighbour is then

$$
\Delta E_{C r}=e_{C r C r} \times n_{C r}+e_{F e C r} \times n_{F e}
$$

similarly for Fe

$$
\Delta E_{\mathrm{Fe}}=e_{\mathrm{FeCr}} \times n_{\mathrm{Cr}}+e_{\mathrm{FeFe}} \times n_{\mathrm{Fe}}
$$

where $e_{\mathrm{CrCr}}, e_{\mathrm{FeCr}}$ and $e_{\mathrm{FeFe}}$ are the energy contributions for each bond of that nature, and $n$ gives the number of nearest neighbours occupied by $\mathrm{Fe}$ or $\mathrm{Cr}$.

For more detail we can look at the work of Chen et al [8] who investigate the migration of $\mathrm{Cr}$-vacancy clusters using the dimer and nudged elastic band (NEB) methods to search for the minimum free energy path. They calculate that a vacancy assisted hop to a 2nd nearest neighbour site has an energy barrier of $2.0 \mathrm{eV}$, which establishes that nearest neighbour migration will be sufficient for our model. Amongst their calculations are values for energy barriers for both $\mathrm{Fe}$ and $\mathrm{Cr}$ migration, with 1-2 vacant nearest neighbours, and up to $2 \mathrm{Cr}$ present in the local environment. From which we assign $e_{\mathrm{FeFe}}=0.094 \mathrm{eV}, e_{\mathrm{FeCr}}=0.08 \mathrm{eV}$, and $e_{\mathrm{CrCr}}=0.01 \mathrm{eV}$, resulting in a regime where $\mathrm{Cr}$ is the more mobile species. The bond energy contribution for $\mathrm{Cr}-\mathrm{Cr}$ bonds is low, so it would be inappropriate to use this for alloys primarily composed of $\mathrm{Cr}$, as the energy barriers would be so low that the applicability of Boltzmann statistics would come into question. However, in our model we are interested in $\mathrm{Cr}$ reaching a threshold 
level of up to $30 \%$, so this is not a concern.

The bond energies chosen above give us a mobile $\mathrm{Cr}$ regime. If we include a surface in the simulation the combination of this higher mobility, and the fact that more events occur at the surface due to the greater number of atoms with vacant neighbours, results in a surface driven $\mathrm{Cr}$ current. We wish to compare the effect of a $\mathrm{Cr}$ current on surface $\mathrm{Cr}$ enrichment and passivation, to a situation where preferential Fe dissolution is the only mechanism. So we also run simulations where all atoms move like $\mathrm{Fe}$, that is $e_{\mathrm{CrCr}}=e_{\mathrm{FeCr}}=e_{\mathrm{FeFe}}=0.094 \mathrm{eV}$, so that the current is removed, but Fe can still be dissolved selectively.

\subsubsection{Three species}

Given that vacancy assisted nearest neighbour migration is the preferential transport mechanism, we required each lattice site to contain one of three species; Fe, $\mathrm{Cr}$ or a vacancy. This requirement entailed a significant generalisation of the original two species KMC code.

Sites are grouped in the list based on their own occupation, and nearest neighbour environment. We determine and update throughout the simulation how many $\mathrm{Fe}, \mathrm{Cr}$, and vacant nearest neighbours a site has, on top of keeping track of that site's own occupation. Given the fixed number of nearest neighbours (periodic boundary conditions were employed), three parameters are required achieve this. Whenever an event occurs, these parameters must be adjusted appropriately, the site moved to the appropriate location on the list, and the list index updated.

For book-keeping purposes all lattice sites are included in the list, even those with no applicable events. For example, we include sites with no vacant nearest neighbours, and therefore no available diffusion sites and also vacancies themselves. We are then easily able to update the local environment at the end of a KMC step, without requiring a list of variable length. 


\subsection{Surface implementation}

We chose the $<110>$ close packed plane as our surface of interest. Introducing a surface added complications to the KMC code. In particular, introducing a surface initially resulted in significant disassociation of atoms from the surface. Also, we needed to keep track of where the surface was located throughout simulations in order to model dissolution.

\subsubsection{Disassociation}

With our bond counting scheme, atoms with the lowest number of solid neighbours have a lesser migration energy barrier $\Delta E$, and therefore are the most likely to move. However, the calculated $\Delta E$ does not include a significant contribution for complete disassociation from the solid. We therefore chose to disallow dissociative migration events, and generate separate rates for dissolution events.

However, disallowing events is subtle because it is dependant on information about the local environment of the final site of a hop. No consideration is given to the final site in the calculation of rates, and no information about it is stored in the list.

We do implement one control over final site selection which mitigates the disassociation effect. Once an event is selected, if there is a choice between a final site with all vacant neighbours and one with at least one solid neighbour, the latter is chosen. However, while this discourages disassociation, it does not eliminate it. Significant disassociation is still observed, as shown in figure 3.3. We can understand this effect given that once an event has been chosen a hop must be made, even if all final sites are disjoint from the surface.

A simple solution is to introduce post selection. That is, after an event has been selected, test the possible final sites. If they are all disjoint from the surface, discard the selection, do not increment time and return to the start of the KMC algorithm. While post selection does not add a large 


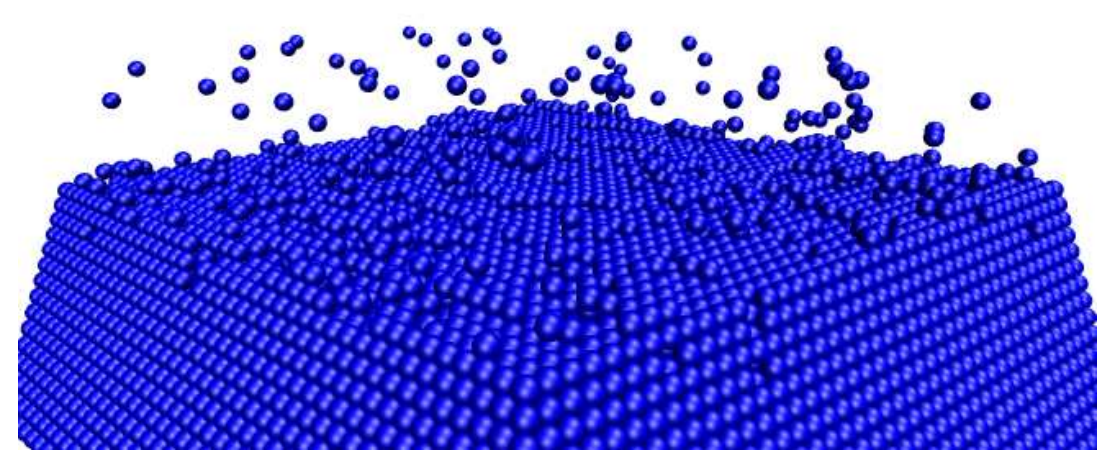

Figure 3.3: Disassociation from surface in pure Fe after $10^{6} \mathrm{KMC}$ steps at $700 \mathrm{~K}$

computational expense, half the number of events selected are discarded. With such large numbers of events discarded our time iteration comes into question. While time is not incremented if an event is discarded, the time step for every event is dependant on the total rate, which includes contributions from a large number of events that we will never allow to occur.

Instead we implement a solution that stops rates being calculated for dissociative migration events. To comprehensively include the final site in the calculation of migration rates would be both impractical and computationally expensive, requiring $72^{2}$ rates to be calculated and arranged in the list.

In practice we do not need comprehensive information about the local environment of the final site. Instead, we designate another type of vacancy which is simply disallowed as a final hop site. We also allow the Fe, and $\mathrm{Cr}$ atoms to take a second type, fixed, rather than mobile.

A vacancy is disallowed if it is isolated from the surface. We could define isolation generally as a vacancy having most nearest neighbours vacant (not all, or it would not be considered as a final site anyway). However, we implement a rule specific to the $<110>$ surface plane, because it helps with the problem of tracking the surface as outlined in the following 
section. If a vacancy has all four of its in - plane nearest neighbours vacant, it becomes disallowed. An atom then becomes fixed if all its nearest neighbour vacancies are disallowed. Note, if an atom has no vacant nearest neighbours, it remains designated as allowed. This avoids atoms in the bulk being flipped from allowed to disallowed unnecessarily. Figure 3.4 shows a 2D cubic lattice representation of these rules.

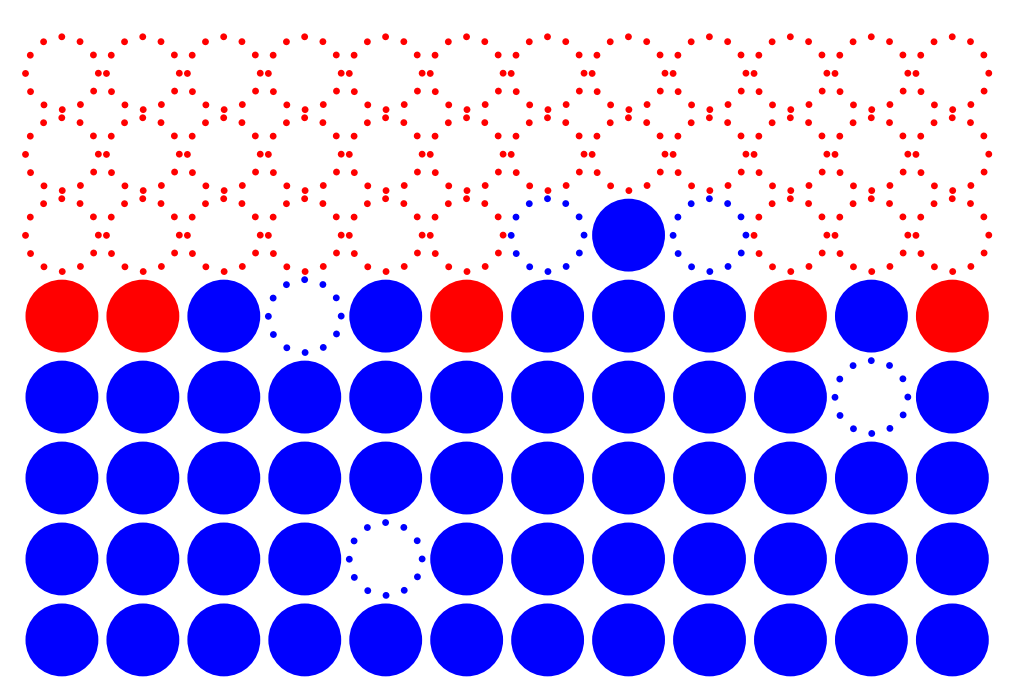

Figure 3.4: 2D cubic lattice representation of disassociation limiting rules. Dashed circles are vacant sites, blue are allowed final hop sites, red are disallowed. Filled circles are atoms; blue are mobile, red are fixed.

A general update determines all disallowed vacancies and fixed atoms at the start of the simulation. No rates are calculated for fixed sites. A local update is then performed in the last step of the KMC algorithm. The local update in itself does not contribute significantly to the simulation time. However, there are now twice as many partitions in the list, which adds to the computational expense of shuffling sites up or down it.

With these conditions in place, vacancies must be introduced to the surface through dissolution, or the metal pre-defected for diffusion to occur. 


\subsubsection{Tracking surface}

First, we need to define which atoms are considered to be at the surface, which is not entirely intuitive with the $<110>$ surface place. In this orientation a surface atom has four nearest neighbours in-plane. While one lattice vector is in-plane, the two remaining lattice vectors are not perpendicular to the surface plane, and therefore neither are the remaining nearest neighbours. We must look to the 5 th nearest neighbour to find one atom directly above another. So, as vacancies are introduced, whether or not an atom is on the surface becomes less well defined.

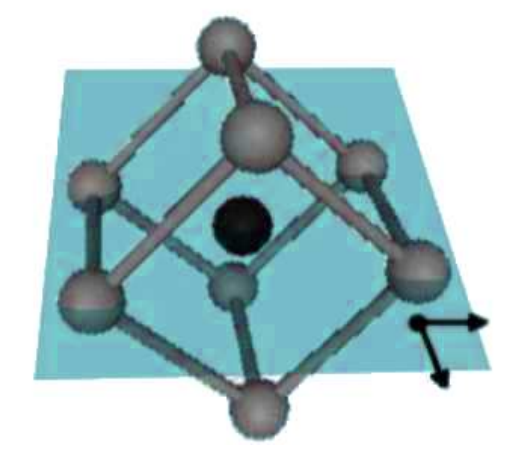

Figure 3.5: BCC cell with a $<110>$ surface plane. No nearest neighbours are perpendicular to the surface plane.

Two arrays could store the uppermost atom in each of the two out of plane directions and thus define the surface. However, this method would present several problems in executing the KMC algorithm. Many sites would be duplicated across the two arrays, so, we would have to ensure such duplications were tracked, so that the counts $C_{n}$ for events were not overstated. Also, if there were deep holes in the surface running along the direction of only one lattice vector, a local update would be insufficient to maintain the surface arrays.

This situation is further complicated for a BCC lattice with vacancy assisted migration. The number of nearest neighbours in a $\mathrm{BCC}$ lattice is 
relatively low (8 compared to 12 in FCC). Given that there are also vacancies in the bulk, it is not sufficient to say that any atom with a large number of vacant nearest neighbours is therefore at the surface. On a defect free $<110>$ surface, there are only 2 vacant nearest neighbours to define the surface. We simulated a bulk system (with periodic boundary conditions in all lattice directions), and the results in figure 3.6, show that even with a low concentration of vacancies, events with two vacant nearest neighbours are common.

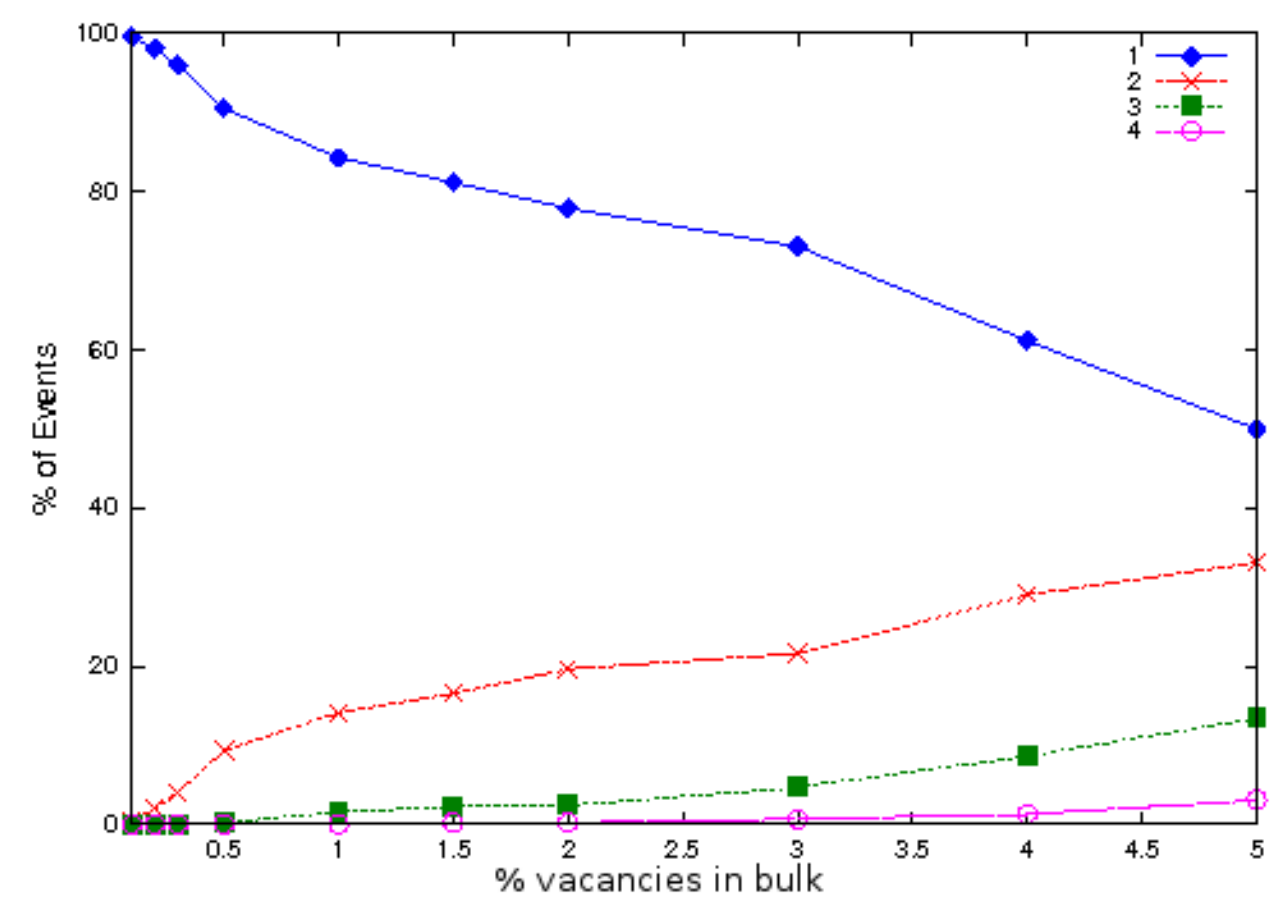

Figure 3.6: Proportion of migration events which occur with a set number of vacant nearest neighbours compared to the bulk concentration of vacancies in pure $\mathrm{Fe}$ at $700 \mathrm{~K}$.

However, the implemented disassociation limiting rules help us here. All atoms that are fixed are at the surface. But, more importantly, almost all atoms with two nearest neighbours at the surface are fixed. This is because the rule for disallowed vacancies is determined by in- plane nearest 
neighbours. We therefore define our surface as all fixed atoms, and all atoms with greater than 3 vacant nearest neighbours are at the surface.

The first limitation of this definition is that atoms under a step edge remain mobile, and are therefore not included in the definition of surface. However, it is reasonable to consider that the mechanism for dissolution in this case is for the atoms to diffuse to the plane of the step edge and then dissolve.

Secondly, the surface must be defined by both nearest neighbours above an atom being vacant. This creates a hole directly above the site. So, an atom with only 1 vacancy above it can not dissolve. We also find this limitation to be acceptable since the probability of an atoms with 7 solid nearest neighbours dissolving is very low.

A more stringent definition of surface, which avoids the first limitation can be employed in cases where we are not limited to storing the information within the list, and therefore the nearest neighbour environment. For example, when writing data about the surface to a file, we can consider beyond the nearest neighbour environment to define the surface, as this information need not be stored in the list. For any atoms that are poorly defined with regard to being on the surface, we check several sites above them, if these sites are vacant, the atom is then classed as being on the surface.

\subsubsection{Dissolution Rates}

Dissolution in Fe-Cr systems is dependant on external factors such as applied potential and $\mathrm{pH}[19,24,29]$. While we have not included any sort of model of the external environment, we have built variable parameters into our model to control dissolution to approximate varying external environments.

A dissolution event removes 1 solid atom from the simulation, and the atom's site becomes vacant. The energy barriers for dissolution events, 
while still dependant on the nearest neighbour environment, were calculated more simplistically than diffusion rates. Only the total number of occupied nearest neighbours was taken into account, rather than their type,

$$
\Delta E=e_{F e F e} \times\left(n_{C r}+n_{F e}\right) .
$$

The reason for this simplification was comparability. We wish to compare the rate of dissolution in alloys with and without a $\mathrm{Cr}$ current. To achieve this we employed the two different bond counting schemes for diffusion, $\mathrm{Cr}$-mobile and Fe-like. If dissolution energy barriers were calculated with the same asymmetry as diffusion in the Cr-mobile regime, $\Delta \mathrm{E}$ could be up to $15 \%$ lower than in the Fe-like regime. So, the time dependence of dissolution in the two schemes would be incomparable.

In calculating the rates for dissolution events, we introduce a variable prefactor $\nu_{1}$ such that

$$
R_{n}=\nu_{1} \nu_{0} \exp \left[\frac{-\Delta E}{k_{b} T}\right]
$$

essentially allowing us to change likelihood of dissolution in relation to diffusion. The prefactor for Fe dissolution and $\mathrm{Cr}$ can be set independently. Primarily $\nu_{1}$ is varied by factors of 10 between $10^{-2}-10^{-5}$ making a dissolution event 100 to $10^{5}$ time less likely than a diffusion event from the same site.

\subsubsection{Passivation}

Formation of the passive oxide film is not modelled explicitly. No oxygen atoms are included, nor have we made any attempt to model the oxide spinel lattice structure. Rather, on the BCC lattice, a condition for passivation is set and implemented. If a particular site becomes passivated, that atom can no longer diffuse or dissolve. This is a reasonable approximation to the early stages of film growth and bond formation. 
We initially wanted to implement something like a nucleation and growth model of the passive film forming, while remembering that the key condition that we wanted to replicate was surface $\mathrm{Cr}$ enrichment. In considering an appropriate condition to apply for passivation, several factors become apparent. Firstly, in considering the local environment of a site for passivation, the nearest neighbour environment is too restrictive. A surface atom will typically have less than 6 nearest neighbours, meaning one $\mathrm{Cr}$ nearest neighbour corresponds to $17 \% \mathrm{Cr}$ in the local environment, and 2 nearest neighbours is $33 \% \mathrm{Cr}$ content in the local environment. This is clearly insufficient resolution to model a sharp onset of passivation. Secondly, if an atom becomes passivated, all atoms in the considered local environment must also be passivated, as if the oxide structure is forming. This should be applied to both surrounding Fe and $\mathrm{Cr}$ atoms. Otherwise, a mobile $\mathrm{Cr}$ could tip many Fe into becoming passive.

Comparison of the BCC lattice with the spinel lattice also illustrates that the nearest neighbour environment is too restrictive. There are no spinel cation neighbours within the same radius as the BCC 1st nearest neighbour distance irrespective of whether we are considering octahedral or tetrahedral sites. The same is true for the 2 nd nearest neighbour distance. We must go to third nearest neighbour distance in the BCC lattice to find an acceptable approximation between the lattices.

Our rule for passivation was then set as follows: if an Fe atom is at the surface, and within the 3rd nearest neighbour distance the concentration of $\mathrm{Cr}$ exceeds a threshold level, the atom and all nearest neighbours within that radius are passivated, they can no longer diffuse or dissolve.

The threshold level of $\mathrm{Cr}$ is worked out as a concentration percentage, rather than a fixed number of $\mathrm{Cr}$ atoms. Otherwise atoms with few neighbours, would never become passivated, even if all these neighbours were $\mathrm{Cr}$. Only Fe atoms are considered in applying the rule for passivation, otherwise two different percentages would be required for $\mathrm{Fe}$, and $\mathrm{Cr}$ for consistency. 
We can then compare this threshold value to first principles electronic structure calculations performed by Ingham et al. [13] who calculated threshold levels of $x$ for thermodynamic stable oxides, $\mathrm{Fe}_{3-2 x} \mathrm{Cr}_{2 x} \mathrm{O}_{4}$, that form on Fe-Cr alloys.

Passivation was not implemented as a stochastic process, hence there are no calculated rates. Rather, passivation is performed as an update after each KMC step. To apply the passivation rule consistently, a check for passivation was required in any cases where $\mathrm{Fe}$ or $\mathrm{Cr}$ migrated within 3rd nearest neighbour distance of a surface Fe atom and also, when Fe dissolved.

In addition, including 2 species that have no associated events provides an easy way to set a fixed boundary condition for the bottom plane in the simulation lattice. This is preferable to having two surfaces in the simulation box, because it allows us to study $\mathrm{Cr}$ migration in a single direction.

\subsection{Summary}

We have developed a kinetic Monte Carlo model which allows for diffusion and dissolution events as well as modelling the formation of a passive film.

Diffusion is dependant on the type of atom and its local environment. Most importantly we have two different diffusion regimes we can compare, one where everything diffuses like Fe and the second where $\mathrm{Cr}$ is more mobile. Dissolution events are determined by the number of solid neighbours and a prefactor which we control independently for different species. This allows us to vary both the likelihood of dissolution compared to diffusion, and also study the impact of $\mathrm{Cr}$ dissolution on Fe dissolution. Surface atoms can become passivated. We can control the threshold percentage of $\mathrm{Cr}$ required in the local environment for passivation to occur. We also control the temperature, simulations are run at fixed temperatures between $300 \mathrm{~K}$ to $700 \mathrm{~K}$ which impacts which events are more likely. Finally 
the bulk concentration of $\mathrm{Cr}$ is varied, typically between $5 \%$ to $25 \%$.

We have a range of parameters that we can vary to investigate their impact on the dissolution of Fe over time, results of which are given in the following section. Table 3.1 gives a summary of the events available for different species.

\begin{tabular}{|c|c|c|}
\hline Type & Represents & Allowed Events \\
\hline 0 & Vacancy & Diffusion to \\
\hline 1 & $\mathrm{Fe} \quad \mathrm{v}<3$ & Diffusion from \\
\hline & $\mathrm{v} \geq 3$ & Diffusion from, Dissolution from \\
\hline 2 & $\mathrm{Cr} \quad \mathrm{v}<3$ & Diffusion from \\
\hline & $\mathrm{v} \geq 3$ & Diffusion from, Dissolution from \\
\hline 3 & Vacancy & None \\
\hline 4 & Surface Fe & Dissolution from \\
\hline 5 & Surface Cr & Dissolution from \\
\hline 6 & Passive Fe & None \\
\hline 7 & Passive $\mathrm{Cr}$ & None \\
\hline
\end{tabular}

Table 3.1: Possible site occupancies and associated events within the KMC code, where 'type' is the numeric descriptor and ' $v$ ' is the number of vacant nearest neighbours 


\section{Chapter 4}

\section{Results and analysis}

This section presents and discusses results of KMC simulations of $\mathrm{Fe}-\mathrm{Cr}$ alloys. We consider the current of $\mathrm{Cr}$ in periodic bulk simulations and in those which include a surface. In the latter case we consider how temperature, $\mathrm{Cr}$ content, and distribution of vacancies, impact on the current. We establish the time dependence of the rate of dissolution in pure $\mathrm{Fe}$ and compare $\mathrm{Fe}-\mathrm{Cr}$ alloys to this baseline. Finally, we observe a threshold for passive film formation, and establish conditions for the level of this threshold. In particular, we consider the impact of the surface $\mathrm{Cr}$ current on passivation.

\subsection{Chromium current}

\subsubsection{Bulk system}

First we consider a bulk alloy. When Fe-Cr alloys are thermally aged, $\mathrm{Cr}$ precipitation occurs, called $\alpha$-phase separation. The $\alpha$-phase precipitate is not pure $\mathrm{Cr}$ but contains 5\%-15\% Fe [4]. This separation occurs if the $\mathrm{Cr}$ content of the alloy is greater than $9 \%$ and above temperatures of $600 \mathrm{~K}$ [32]. Simulations that have successfully described $\alpha$-phase separation perform a full electronic relaxation every time step, a significant 
computational expense [31, 32, 4]. Bonny et al [4] calculate $10^{10}$ computational steps requiring on the order of 1 month of computational time. Their simulations contain 128000 atoms at $700 \mathrm{~K}$ giving approximately $14 \mathrm{~s}$ simulated time for Fe-Cr alloys containing 12-18\% $\mathrm{Cr}$ and are able to determine the onset of precipitate formation. A comparable calculation using our KMC model requires only 4 days of simulation time, but is unable to resolve the onset of $\mathrm{Cr}$ precipitation. With 30s of simulated $\mathrm{Fe}_{85} \mathrm{Cr}_{15}$ with 132650 atoms (and 1 vacant site) we see no increase in the number of $\mathrm{Cr}-\mathrm{Cr}$ bonds, indicating no general aggregation of $\mathrm{Cr}$.

The limitation is our linear bond counting scheme. Given the nearest neighbour limitation, it cannot account for long range interactions as $\mathrm{Cr}$ groups together. However, the time scale for significant $\alpha$-phase separation is on the order of weeks to months [22], much greater than the time scales for passive film formation. Even the initial onset time of seconds is longer than the timescales we model for the onset of passivation, which are on the order of ms.

One bulk effect we do need to be conscious of in our simulations is that if the $\% \mathrm{Cr}$ is low and the percentage of vacancies is high $(>5 \%)$, vacancies tend to aggregate. Figure 4.1 illustrates this aggregation in a bulk simulation with $10 \%$ of lattice sites vacant, after $7 \mu$ s of simulation time significant aggregation of vacancies is observed. This effect quickly decreases below $5 \%$ vacancy content, and is indistinguishable at $2 \%$. Figure 3.6 in the methods section reiterates this point. Below 3\% vacancy content, there are very few events with more than 3 vacant nearest neighbours, indicating that vacancies are remaining well mixed in the system. However, in simulations that include dissolution we must still keep track of the amount of vacancies introduced to the bulk. In particular, if many vacancies are introduced via dissolution, and aggregation occurs, our definition of surface atoms could break down. Typically though, vacancies introduced via dissolution number on the order of $10 \%$ of the number in a surface plane. This equates to $0.25 \%$ vacancies in the bulk, well below the level required 


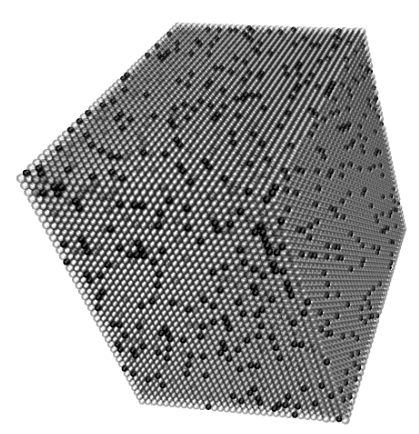

(a)

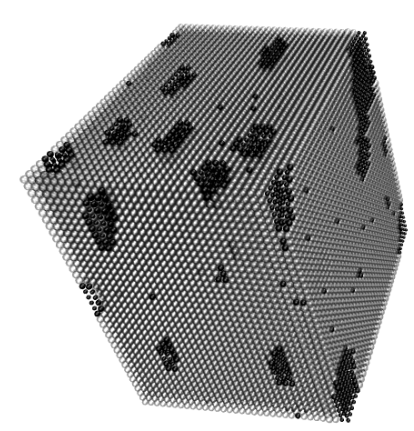

(b)

Figure 4.1: Pure Fe with $10 \%$ of lattice sites vacant. Vacancies are shown in black (a) initial random distribution (b) final distribution of vacancies after $7 \mu \mathrm{s}$, aggregation of vacancies is observed.

for vacancy aggregation.

\subsubsection{Surface system}

We model a close-packed nearest surface plane containing 1681 atoms, which has an area of $110 \mathrm{~nm}^{2}$. The block has a depth of 34 atomic planes or $90 \mathrm{~nm}$. The initial distribution of atoms is random. A random number, $r$, on the range $(0,1)$ is drawn for each lattice site, if that number is less than $\phi$ the site is occupied by $\mathrm{Cr}$, otherwise $\mathrm{Fe}$, with the exception of the surface plane. To emulate the introduction of vacancies by dissolution $10 \%$ of sites in the surface plane are initially vacant. Again occupation is selected by random number draw for each site. If $\mathrm{r}<0.1$ the site is vacant, and if $\mathrm{r}$ $<0.1+0.9 \phi$ the site is occupied by $\mathrm{Cr}$, otherwise Fe. This maintains the ratio of $\mathrm{Fe}$ to $\mathrm{Cr}$ in the surface plane. There are in total 56,986 atoms in the simulation, and approximately 168 vacant sites available for vacancy 
assisted diffusion.

In modelling a surface rather than a bulk system, we observe a current of $\mathrm{Cr}$ towards the surface. Figure 4.2 shows the final distribution of $\mathrm{Cr}$ in alloys with $5 \%$ to $25 \%$ chromium content at temperatures $300 \mathrm{~K}, 500 \mathrm{~K}$, and $700 \mathrm{~K}$. The proportion of $\mathrm{Cr}$ in each plane parallel to the surface is plotted. All curves in figure 4.2 are shown after $10^{7}$ computational steps rather than at a fixed time, in order to illustrate the computational barriers to simulating at varying temperatures.

We first observe that in the majority of cases the proportion of $\mathrm{Cr}$ is significantly enriched in the surface plane compared to the random distribution in the rest of the block. For example, at $700 \mathrm{~K}$ there is at least $10 \%$ more $\mathrm{Cr}$ in the surface plane. The second plane from the surface is depleted, indicating a significant amount of the additional surface $\mathrm{Cr}$ originated from there.

Given that $\mathrm{Cr}$ was randomly distributed in the initial configurations, it is clear that $\mathrm{Cr}$ has moved to the surface. This current results from the combination of the higher mobility of $\mathrm{Cr}$ atoms, and the number of events which occur at the surface. Since all atoms at the surface are in contact with vacancies, there are more atoms with low energy barriers at the surface, and therefore more events occur there. Secondly, if an atom moves from the second plane to the surface plane it is more likely to be a $\mathrm{Cr}$ atom, because the energy barriers for $\mathrm{Cr}$ diffusion are lower than for Fe.

This surface enrichment of $\mathrm{Cr}$ occurs irrespective of whether vacancies are introduced at the surface, or distributed randomly through the bulk. Though, the process starts more slowly in the latter case. The simulations in figure 4.2 have $10 \%$ of lattice sites in the surface plane vacant emulating the introduction of vacancies through dissolution. The migration of $\mathrm{Cr}$ is to the surface plane is sharp, as shown in Figure 4.3, for $\mathrm{Fe}_{0.85} \mathrm{Cr}_{0.15}$ at $700 \mathrm{~K}$. The vacant sites quickly propagate into the bulk, and $\mathrm{Cr}$ atoms primarily fill their places. Figure 4.3 also shows the progression of $\mathrm{Cr}$ build up when the same total number of vacancies are distributed randomly throughout 


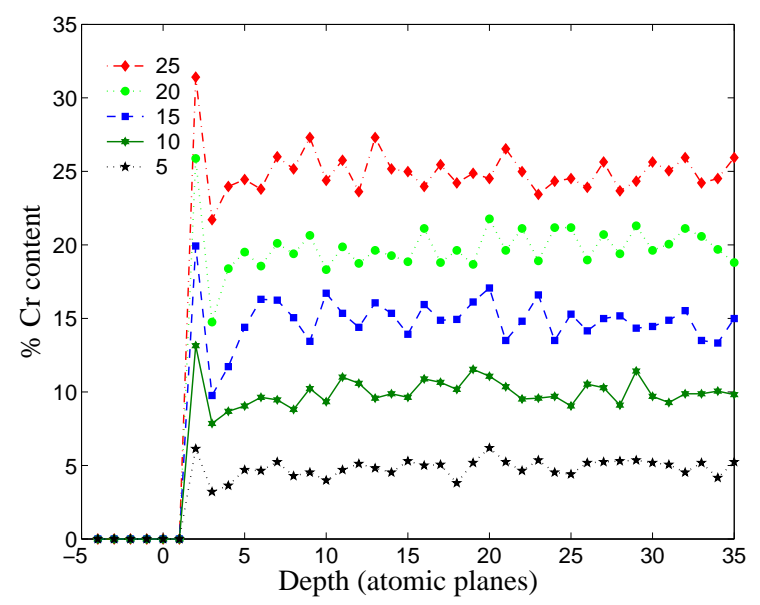

(a)

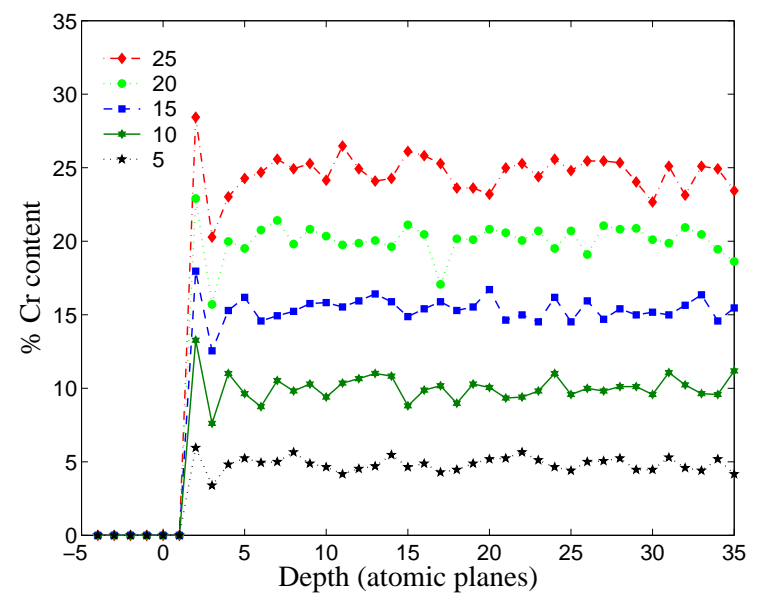

(b)

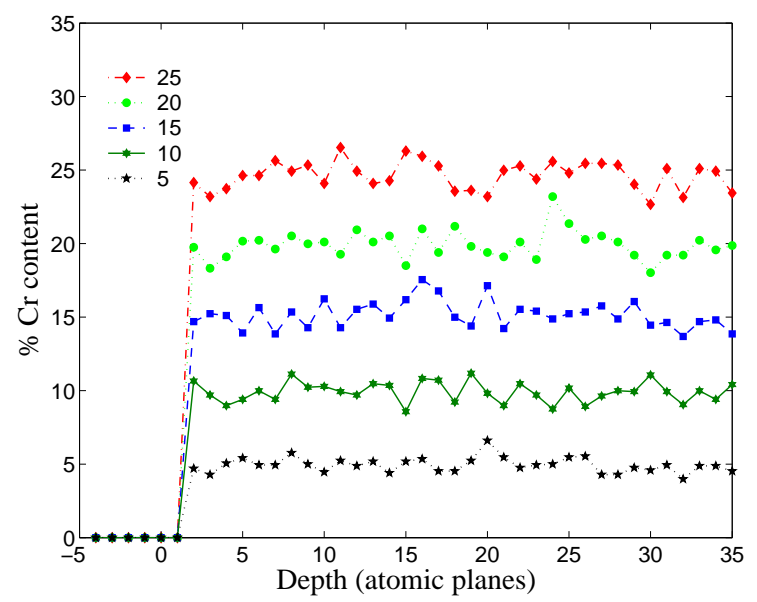

(c)

Figure 4.2: Distribution of $\mathrm{Cr}$ in planes parallel to the surface after $10^{7}$ computational steps at temperatures (a) $700 \mathrm{~K}$ (b) $500 \mathrm{~K}$ (c) $300 \mathrm{~K}$. The legend gives the percentage of $\mathrm{Cr}$ in the alloy. $41 \times 41 \times 34$ block 
the bulk. In this case the surface build of $\mathrm{Cr}$ is slower, when vacancies migrate to and from the surface, they allow for the gradual redistribution of $\mathrm{Cr}$ there.

Now let us consider temperature effects. At varying temperatures, different events become accessible to the system. Cr migration has lower energy barriers, so is the more prevalent event. More so, $\mathrm{Cr}$ migration is proportionally more prevalent at lower temperatures. So in $\mathrm{Fe}_{0.75} \mathrm{Cr}_{0.25}$ at $700 \mathrm{~K}, 4 \%$ of events are Fe migration, at $500 \mathrm{~K}$ this drops to $1.6 \%$. The effect is more pronounced when there is lower $\mathrm{Cr}$ content in the alloy as more $\mathrm{Fe}$ in the system results in more $\mathrm{Fe}$ events. So in $\mathrm{Fe}_{0.95} \mathrm{Cr}_{0.05}$ at $700 \mathrm{~K}, 60 \%$ of events are Fe migration, which drops to $39 \%$ at $500 \mathrm{~K}$. At $300 \mathrm{~K}$ nearly all events are $\mathrm{Cr}$ migration. Only $0.5 \%$ with $75 \%$ Fe content, and $14 \%$ with 95\% Fe content, of events are Fe migration. Rather than enriching the surface more rapidly, the process seems to be inhibited. So, interestingly some Fe diffusion is required to allow $\mathrm{Cr}$ build up at the surface. A possible reason is that the same $\mathrm{Cr}$ atoms are moving back and forth into the same vacancies. This idea is supported by the lack of vacancies which propagate into planes below the surface at $300 \mathrm{~K}$. At $300 \mathrm{~K}$, when the $\mathrm{Cr}$ content is below $15 \%$, no vacancies propagate below the surface plane. The same is true at $700 \mathrm{~K}$ but, only at $5 \% \mathrm{Cr}$ content. However, in $\mathrm{Fe}_{0.75} \mathrm{Cr}_{0.25}$ at $300 \mathrm{~K}$ only 5 vacancies of 170 are found below this plane and do not reach a depth lower than the 3 rd plane. Compare this to $700 \mathrm{~K}$, where 38 vacancies spread to the furthermost plane from the surface in $\mathrm{Fe}_{0.75} \mathrm{Cr}_{0.25}$.

So, at lower temperatures, similar patterns of surface $\mathrm{Cr}$ build up are occurring. But, more computational steps are required to model the current. For this reason simulations in the following sections are performed at $700 \mathrm{~K}$.

Finally let us consider the time scales over which this flow to the surface is occurring. At $700 \mathrm{~K}$ simulations cover between $19 \mu$ s for $\mathrm{Fe}_{0.75} \mathrm{Cr}_{0.25}$ to $150 \mu \mathrm{s}$ for $\mathrm{Fe}_{0.95} \mathrm{Cr}_{0.05}$. So, at a set physical time the difference with increasing surface chromium enrichment will be more pronounced than in 


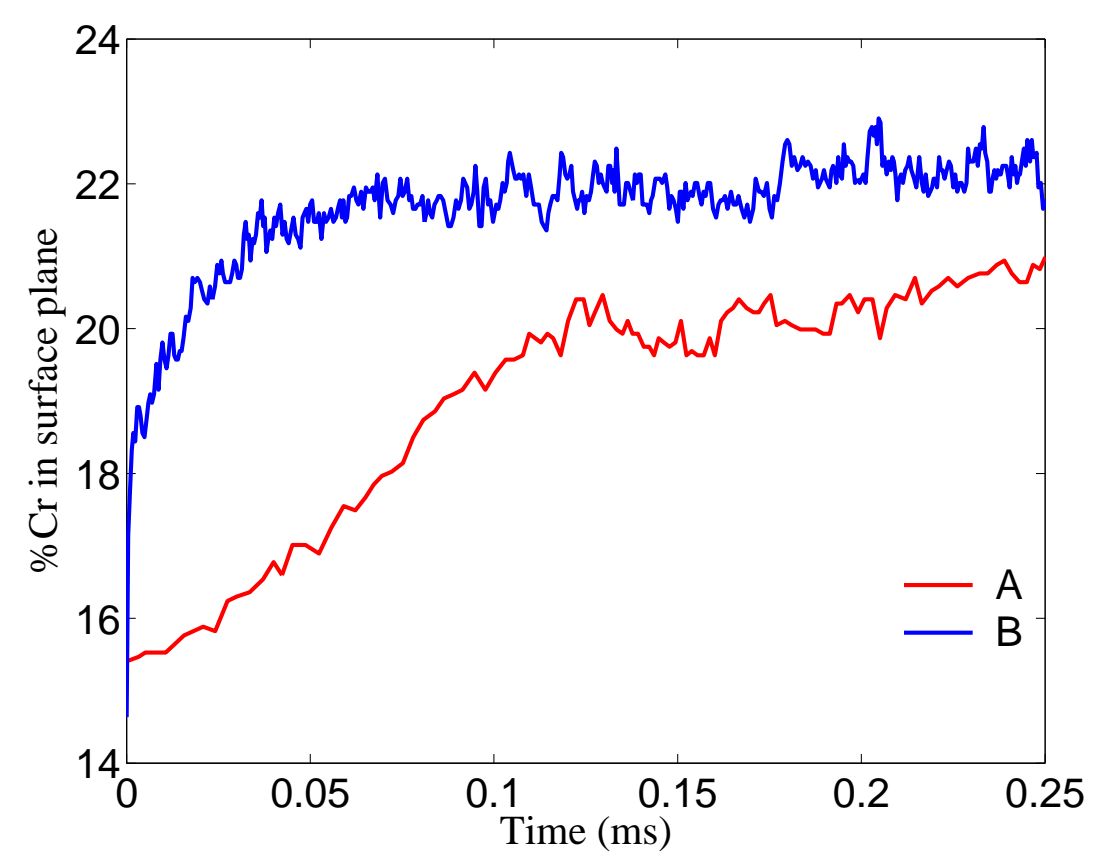

Figure 4.3: Evolution of $\% \mathrm{Cr}$ in the surface plane of an $\mathrm{Fe}_{0.85} \mathrm{Cr}_{0.15}$ alloy over time at $700 \mathrm{~K}$ when in the initial configuration (a) $10 \%$ of surface sites are vacant (b) the same number of vacancies are spread throughout the alloy 
figure 4.2, indicating the current of $\mathrm{Cr}$ to the surface is slower with decreased $\mathrm{Cr}$ content.

At $700 \mathrm{~K}$ on a longer time scale, $1 \mathrm{~ms}$, the surface current slows. The surface $\mathrm{Cr}$ enrichment appears to reach a maximum level around $40 \%$. Suzuki et al. [28] have experimentally observed the enrichment of $\mathrm{Cr}$ at the surface. They estimate the thickness of the segregated zone as less than 1 $\mathrm{nm}$. Our atomic plane thickness is $0.24 \mathrm{~nm}$. They also found that the segregated area contains roughly 3 times the content of $\mathrm{Cr}$ in the alloy, $45 \%$ for an alloy containing $13 \% \mathrm{Cr}$, and $69 \%$ for $25 \%$. So, while our model doesn't entirely reproduce this variation in surface enrichment, it is particularly accurate for lower alloy $\mathrm{Cr}$ contents, where we expect to observe changing passivation behaviour.

In summary, while our bond counting scheme is too simplistic to model phase separation in the bulk, introducing a surface allows us to simulate a surface driven $\mathrm{Cr}$ current. It is important to note though, that while the current increases with higher $\mathrm{Cr}$ content in the alloy, there is no onset of this current at a particular \%Cr. However, we want to establish the impact of a $\mathrm{Cr}$ current on surface $\mathrm{Cr}$ enrichment. In particular, we want to compare this mechanism to enrichment resulting purely from preferential Fe dissolution. So, if we allow all the atoms to move like Fe atoms, by removing the asymmetry in the bond counting scheme, we can turn off the $\mathrm{Cr}$ current. $\mathrm{Cr}$ atoms are then no more mobile than $\mathrm{Fe}$, and the current no longer occurs. If we still differentiate between $\mathrm{Fe}$ and $\mathrm{Cr}$ for dissolution events, we then have a viable comparison between mechanisms of surface Cr enrichment.

\subsection{Dissolution}

In the following section we will further investigate the Cr current and surface $\mathrm{Cr}$ enrichment when the method of vacancy introduction is dissolution. 


\subsubsection{Pure Fe}

In modelling the onset of passivation, we are interested in any changes in the progression of Fe dissolution over time, so, we need a baseline of dissolution behaviour for comparison. Here we simulate a pure iron system to establish the pattern of dissolution in a system where there is no passivation occurring. Again, the initial dimensions of the system are 34 atomic planes, each containing 1681 atoms. Here, all atoms are Fe, and there are initially no vacancies in the block.

To study dissolution over time we consider the cumulative number of Fe atoms that have dissolved and the rate of dissolution. Figure 4.4 plots the total dissolved Fe over time for two values of $\nu_{1}, 10^{-2}$ and $10^{-4}$, meaning dissolution is 100, and 10000 less likely than diffusion. In both cases, there is a consistent linear increase in the amount of dissolved Fe, with a repetitive oscillation around this trend. The linear trend continues until the remaining undissolved material in the simulated block becomes limited. We observe no overall time dependence to the rate of dissolution.

The oscillations are a size effect related to the number of atoms in the simulated surface plane. There are 1681 atoms in each plane parallel to the surface. As seen in figure 4.5, dissolution is primarily proceeding one plane at a time, starting more slowly when there are few vacancies in the surface plane. Then, proceeding most quickly when there are many atoms on the surface with more than 2 vacant nearest neighbours.

The oscillations are less pronounced when dissolution is more likely. In the case where dissolution is much quicker $\nu_{1}=10^{-2}$ dissolution is occurring in several planes at a time.

Let us compare our simulated rate of Fe dissolution to experimental results. Bessone et al [1] measure the maximum current density of Fe cations dissolving in a $\mathrm{pH} 4-5.5$ solution as $1 \mathrm{Acm}^{-2}$. This corresponds to a dissolution rate of $\sim 3 \times 10^{20}$ dissolutions s${ }^{-1} \mathrm{~m}^{-2}$. Over the area considered in our simulations $\left(\sim 100 \mathrm{~nm}^{2}\right)$ this gives a $\sim 3 \times 10^{4}$ dissolutions $\mathrm{s}^{-1}$. With $\nu_{1}=10^{-4}$ in our simulations the dissolution rate is 2 orders of magnitude 


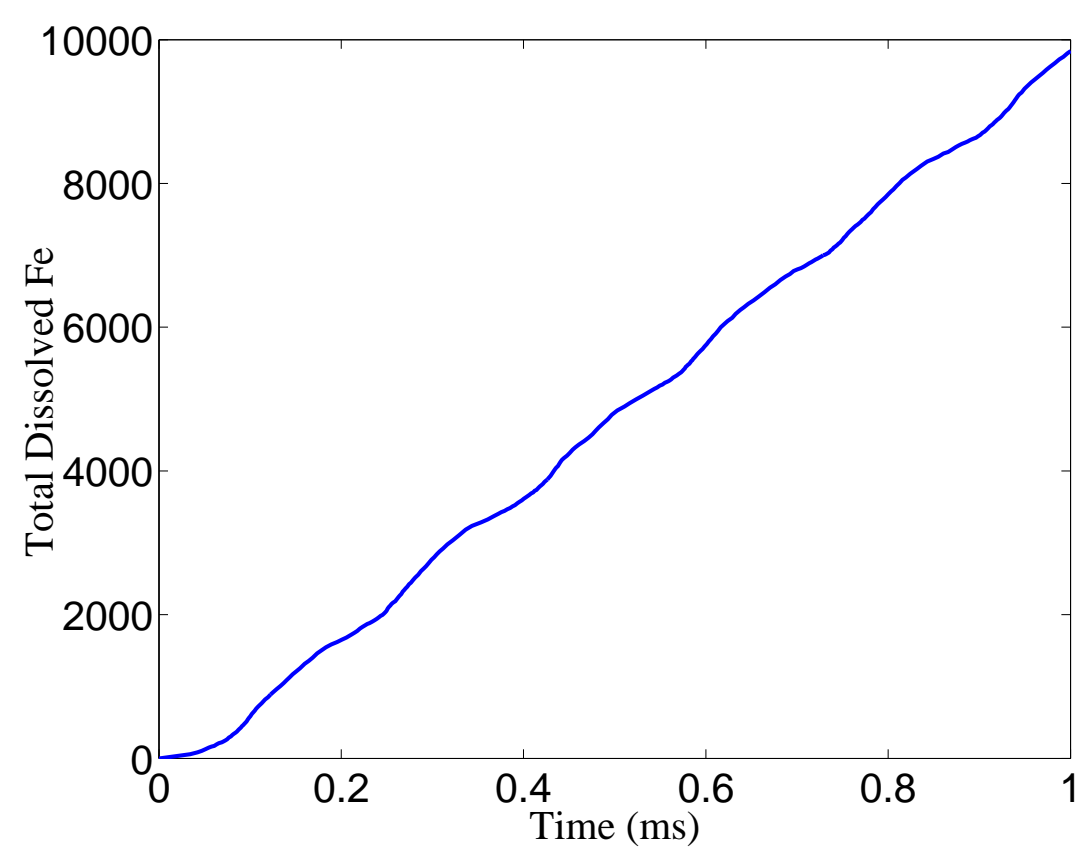

(a)

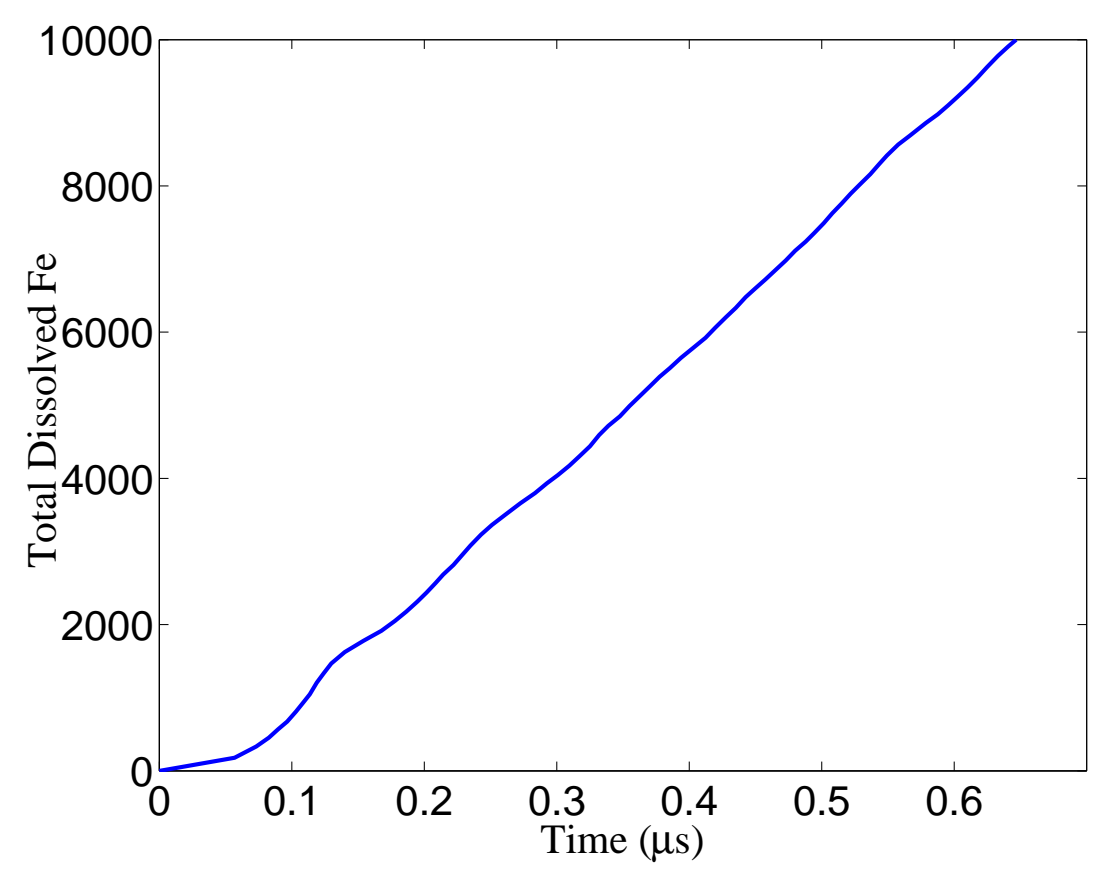

(b)

Figure 4.4: Total dissolved Fe in a pure Fe lattice containing 34 planes parallel to the $\langle 110\rangle$ surface, each containing 1681 atoms (a) $\nu_{1}=10^{-4}$ (b) $\nu_{1}=10^{-2}$ 


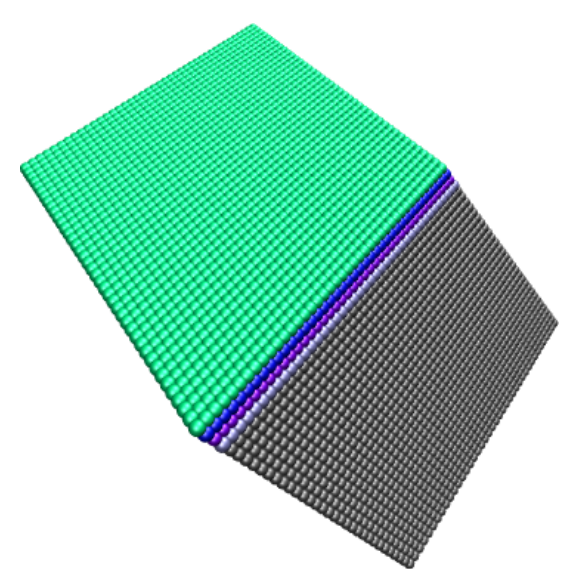

(a)

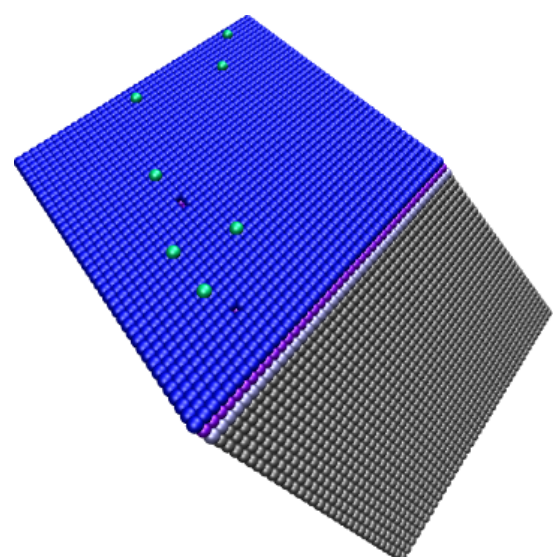

(c)

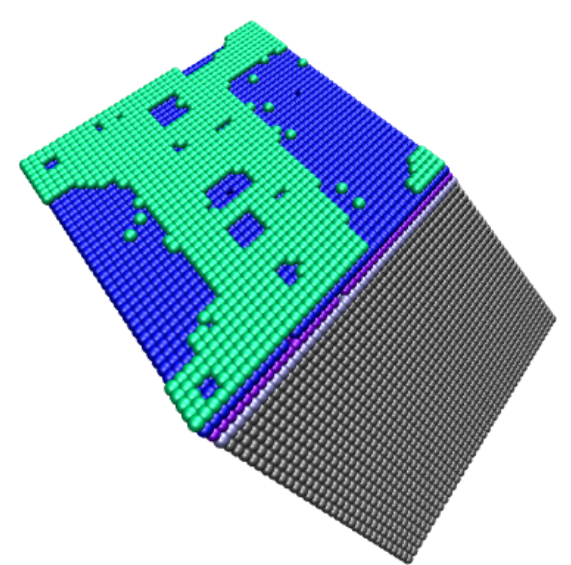

(b)

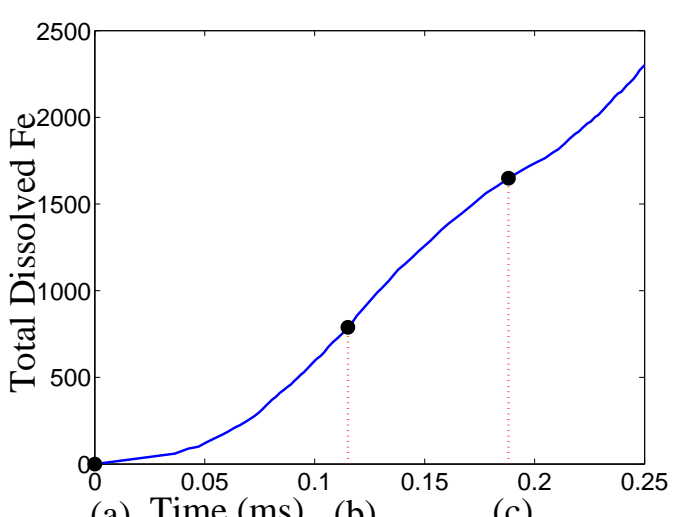

(a) Time (ms) (b) (c)

(d)

Figure 4.5: Dissolution in pure Fe at 700K with $\nu_{1}=10^{-4}$ at (a) $t=0$, no dissolved Fe (b) $t=1.2 \mathrm{~ms}$, the number of dissolved Fe atoms is equal to half the total number in a plane (c) $t=1.9 \mathrm{~ms}$, the number of dissolved Fe atoms is equal to the total number in a plane. The block has 34 planes parallel to the $<110>$ surface, each containing 1681 atoms 
larger, $\sim 10^{6}$ dissolutions $\mathrm{s}^{-1}$. Consider though that these experiments were conducted at $333 \mathrm{~K}$. Our simulation is at $700 \mathrm{~K}$, at lower temperatures diffusion and dissolution rates slow, as does the Cr current. So, while we conduct simulations at $700 \mathrm{~K}$, we will consider trends as $\nu_{1}$ is lowered from $10^{-3}$ to $10^{-5}$.

A final point to note is that significant dissolution limits the time scales that can be reached in simulations. The associated rates are short, diffusion is unlikely, and consequently the time associated with each computational step is short.

\subsubsection{Alloys}

Now we can investigate the time dependence of Fe dissolution in $\mathrm{Fe}-\mathrm{Cr}$ alloys compared to the baseline behaviour in pure iron. We again look at a block with the same dimensions, 34 atomic planes of area $110 \mathrm{~nm}^{2}$, and no initial vacancies. Initially we allow only Fe dissolution.

We find that, even with passivation turned off, the rate of dissolution decreases over time. This effect is due to the proportion of $\mathrm{Cr}$ at the surface increasing. There are less possible Fe atoms to dissolve from the surface, so, the probability of dissolution occurring is lowered.

Figure 4.6 compares the total Fe dissolution over time in an $\mathrm{Fe}_{0.85} \mathrm{Cr}_{0.15}$ alloy to pure Fe. In both the Fe-like and Cr-mobile regimes there is a decrease in the rate of dissolution over time, indicating that surface $\mathrm{Cr}$ enrichment occurs in both cases. However, the change is more significant in the Cr-like regime, indicating a significant $\mathrm{Cr}$ surface enrichment resulting from the $\mathrm{Cr}$ current rather than preferential Fe dissolution. Less dissolution occurs in the $\mathrm{Cr}$-mobile regime for a particular level of surface enrichment to be achieved. This is significant when considering experimental conditions where $\mathrm{Cr}$ surface enrichment occurs with little or no Fe dissolution [19]. The triangles in figure 4.6 show the point where the percentage of $\mathrm{Cr}$ surface atom has increase from $15 \%$ to $23 \%$. One third as 


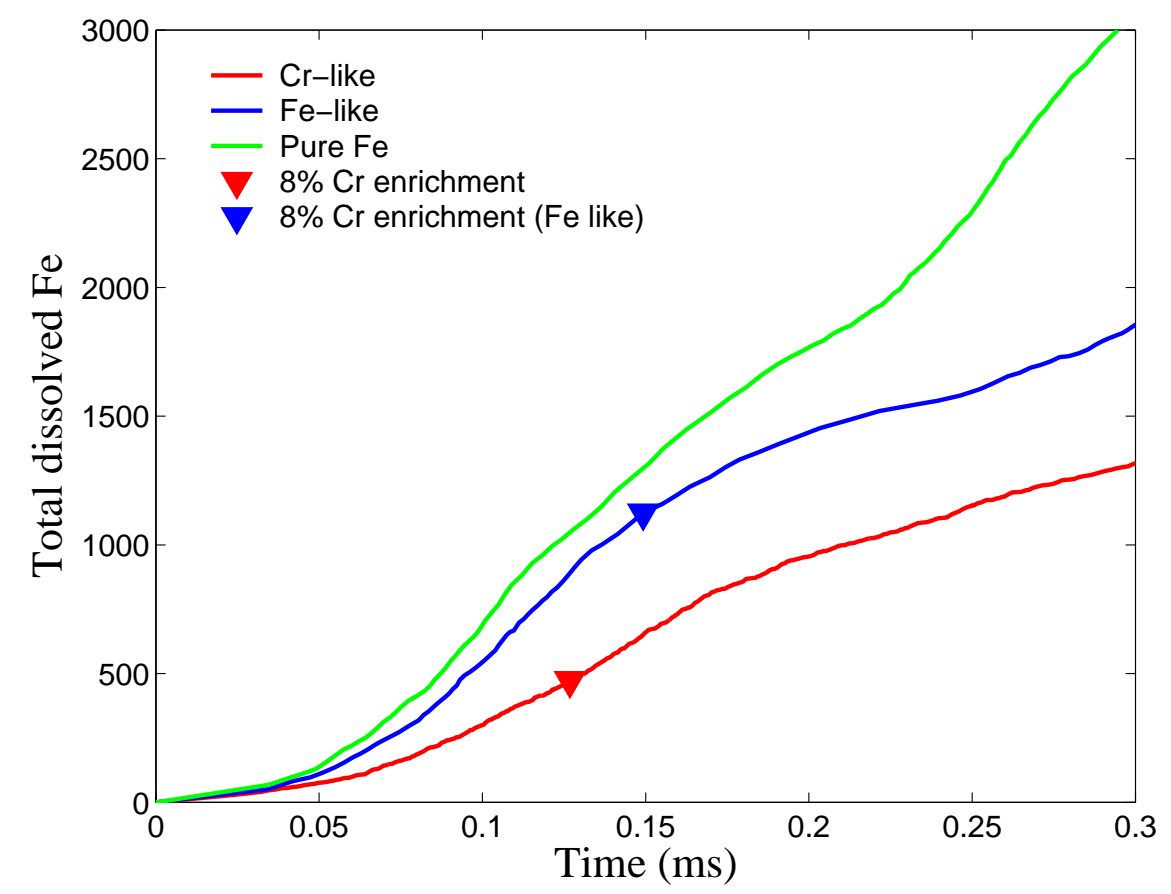

Figure 4.6: Total Fe dissolution over time in pure $\mathrm{Fe}$ and $\mathrm{Fe}_{85} \mathrm{Cr}_{15}$ with and without $\mathrm{Cr}$ current at $700 \mathrm{~K}$. The triangles show the point at which surface $\mathrm{Cr}$ content has increased from $15 \%$ to $23 \%$. The simulation block is 34 planes parallel to the $<110>$ surface, each containing 1681 atoms 


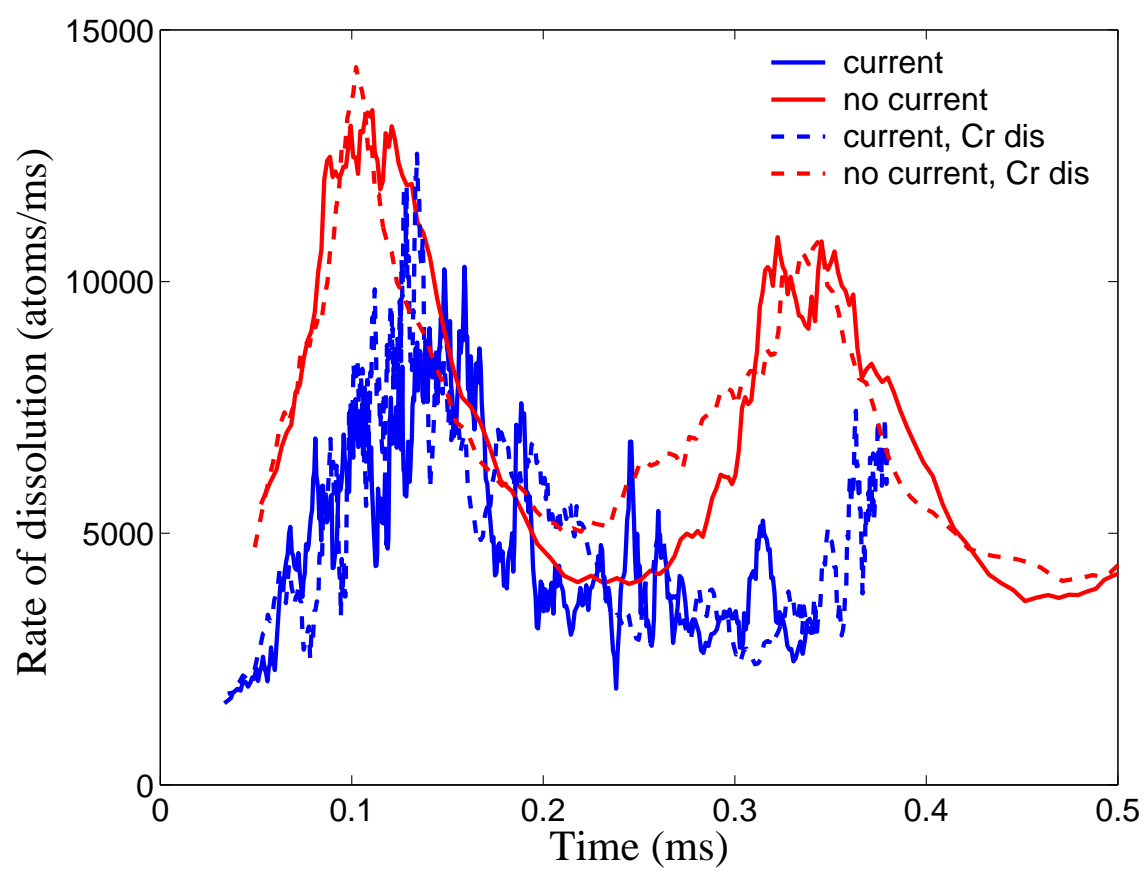

Figure 4.7: Rate of Fe dissolution at $\nu_{1}=10^{-4}$, with and without Cr current, only Fe dissolution, and Fe dissolution as well as $\mathrm{Cr}$ dissolution at $\nu_{1}=10^{-5}$, at $700 \mathrm{~K}$ with 34 atomic planes containing 1681 atoms.

many Fe dissolutions have occurred at this point with the $\mathrm{Cr}$ current compared to without, showing a $\mathrm{Cr}$ current could be appropriate for situations where passivation occurs with minimal Fe dissolution.

We can convert the total Fe dissolution over time into a dissolution rate, as plotted in figure 4.7. It is clear that the rate of dissolution is not constant with time. Also plotted is the Fe dissolution when $\mathrm{Cr}$ dissolution is allowed at a rate 10 times less than diffusion.It is interesting to note that with no $\mathrm{Cr}$ current, $\mathrm{Cr}$ dissolution hastens Fe dissolution, particularly in the trough where the rates are lowest. More vacant neighbours left by $\mathrm{Cr}$ dissolution allows more Fe dissolution. The same effect is not seen in the $\mathrm{Cr}$-mobile regime, perhaps indicating that instead $\mathrm{Cr}$ is diffusing from below to fill these vacancies. In both cases the rate of surface $\mathrm{Cr}$ enrichment 


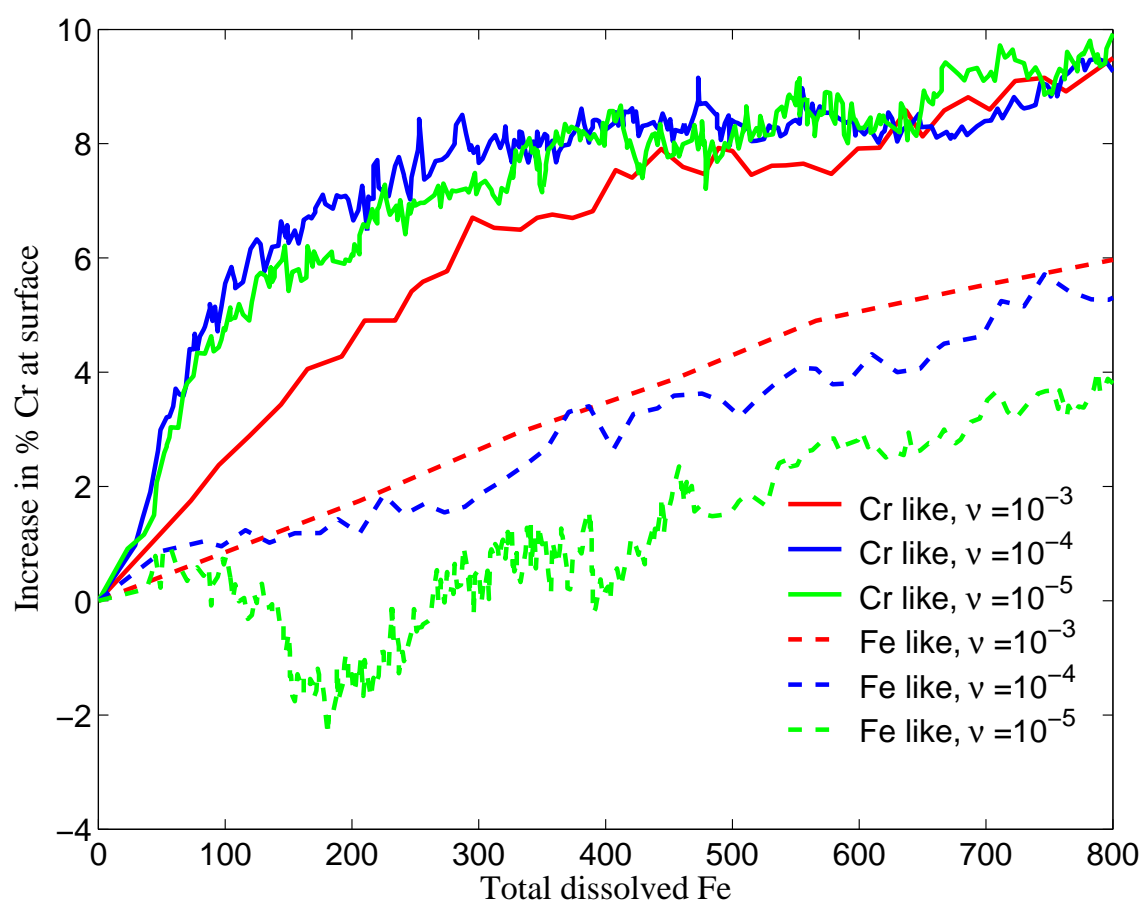

Figure 4.8: Surface $\mathrm{Cr}$ enrichment in $\mathrm{Fe}_{85} \mathrm{Cr}_{15}$ at varied $\nu_{1}$ with and without Cr current at $700 \mathrm{~K}$ in a $41 \times 41 \times$ simulation block.

is slowed marginally with $\mathrm{Cr}$ dissolution. It is interesting that this is the case in both situations, even though the dissolution rate is little changed with the Cr current in place. The increased rate of dissolution seems to help reach the required amount of Fe dissolution for surface enrichment more quickly compensating somewhat for the loss of $\mathrm{Cr}$ itself.

To compare the effect of the set rate of dissolution on surface $\mathrm{Cr}$ enrichment, figure 4.8 plots the increase in the percentage of the surface that is $\mathrm{Cr}$ against the total number of dissolved $\mathrm{Fe}$, for $\mathrm{Fe}_{0.85} \mathrm{Cr}_{0.15}$. This avoids the vastly differing timescales for dissolution, although the data does become noisy when the rate of dissolution is slow. Several features are of interest.

Firstly, consider the data for the Fe-like regime. The surface $\mathrm{Cr}$ content increases approximately linearly with the amount of dissolved Fe. This is explained by preferential Fe dissolution being the only mechanism for 
surface enrichment. An Fe atom must dissolve for the proportion of $\mathrm{Cr}$ at the surface to increase.

In the $\mathrm{Cr}$-mobile regime a more dramatic increase is seen. The $\mathrm{Cr}$ current allows the surface to become enriched with less Fe dissolution. The rate of increase is greatest initially. Only 100 atoms dissolve for an increase from $15 \% \mathrm{Cr}$ content to $20 \%$ compared to 800 atoms without the Cr current.

Finally let us consider variation with $\nu_{1}$. In the Fe-like regime the curves for $\nu_{1}=10^{-3}$ and $\nu_{1}=10^{-4}$ are similar. However, at $\nu_{1}=10^{-5}$ there is much more noise, due to the extended time scale between each dissolution event. The surface $\mathrm{Cr}$ content, in fact, decreases by $2 \%$ before increasing linearly.

In the Cr-mobile regime, there is little difference between the curves at $\nu_{1}=10^{-4}$ and $\nu_{1}=10^{-5}$. However, when $\nu_{1}=10^{-3}$ the rate of enrichment is considerably slower, indicating that dissolution is occurring too quickly for the $\mathrm{Cr}$ current to enrich the surface and slow the rate of dissolution as effectively. The relative time scales of the $\mathrm{Cr}$ current and $\mathrm{Fe}$ dissolution therefore determine how significant the $\mathrm{Cr}$ current is to surface enrichment.

\subsection{Passive film behaviour}

Now we introduce surface passivation. Here, we illustrate that our model shows a clear threshold chromium content for a passive film to form, and investigate the impact of the $\mathrm{Cr}$ current and rate of dissolution on the level of this threshold.

Figure 4.9 shows the total corroded Fe over time for alloys with bulk $\%$ Cr varying from $5 \%$ to $25 \%$. The condition for the formation of the passive film is set at $20 \%$. There is a clear sharp transition in behaviour with increased bulk $\mathrm{Cr}$ content. At $8 \% \mathrm{Cr}$ content and below dissolution is continuous. However, with more than $10 \% \mathrm{Cr}$ content, after an initial period 


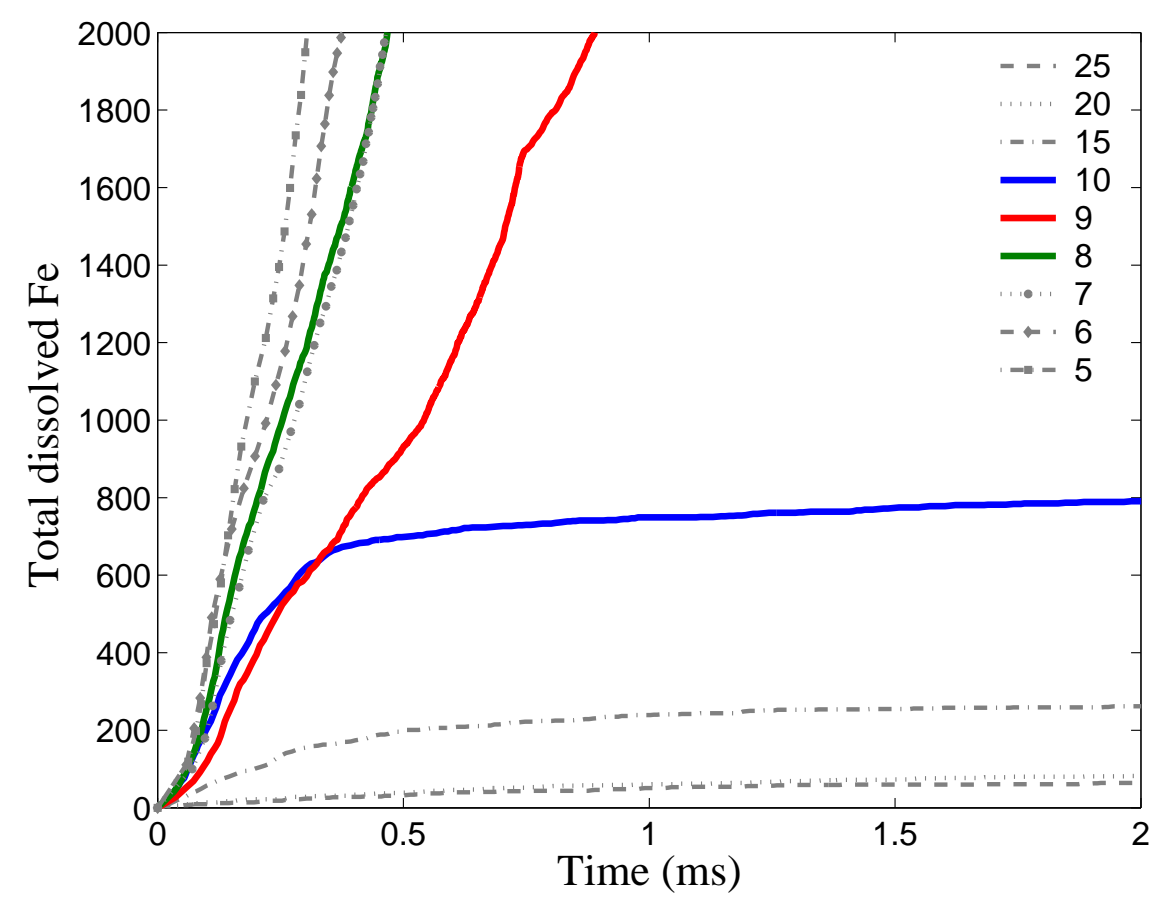

(a)

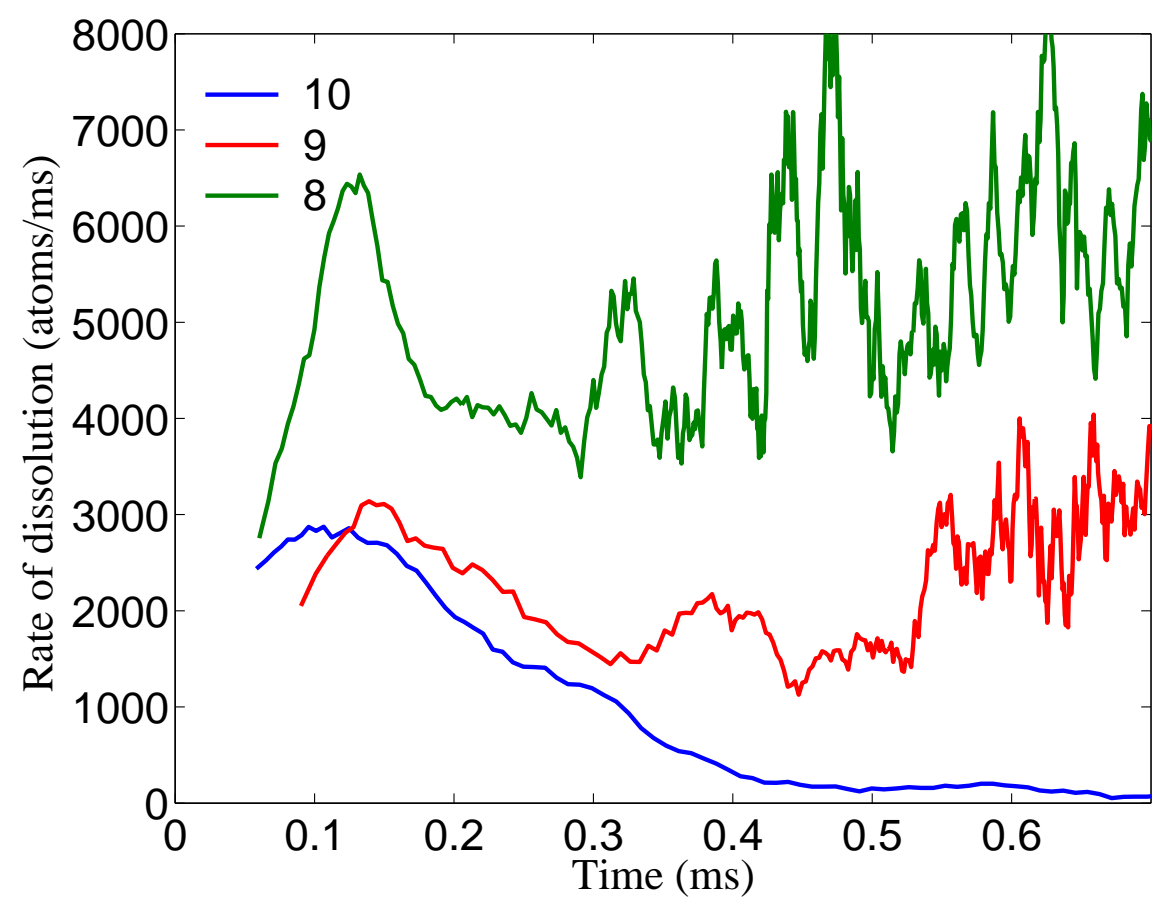

(b)

Figure 4.9: (a) Total Fe dissolution and (b) dissolution rate over time in alloys for bulk Cr content varied from $5 \%$ to $25 \%$ with the local environment threshold for passivation set at $20 \% \mathrm{Cr}$, at $700 \mathrm{~K}$ with 34 planes parallel to the surface containing 1681 atoms each. A clear change in behaviour is observed between $8 \%$ to $10 \%$ bulk $\mathrm{Cr}$ content. 
of dissolution, the rate of dissolution slows and ceases.

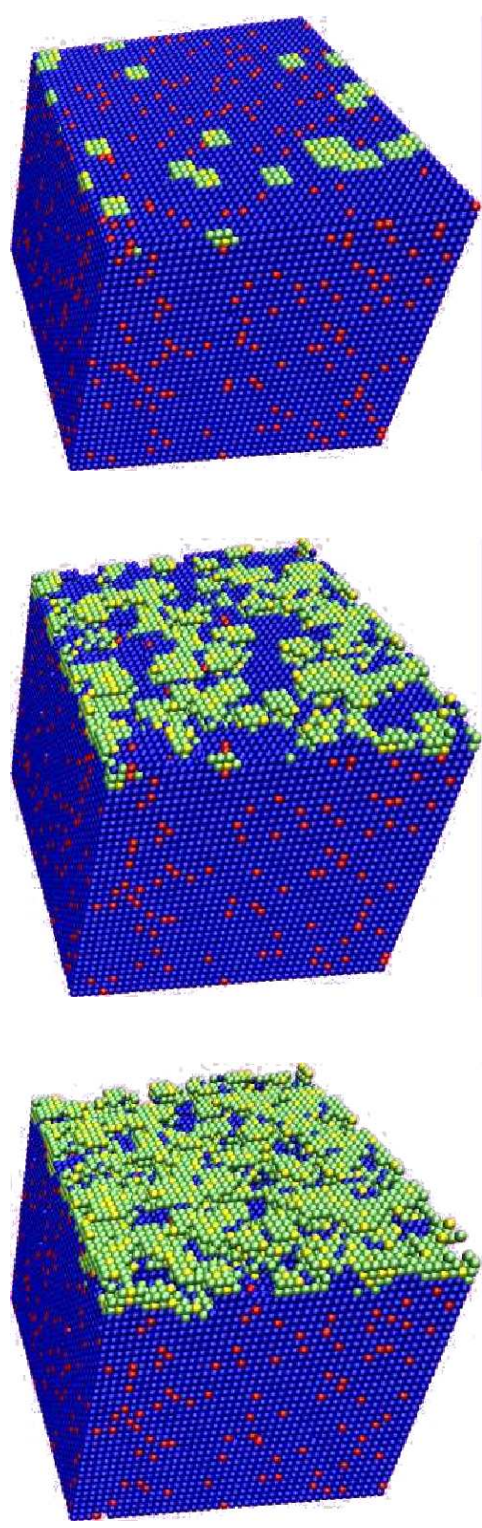

(a)
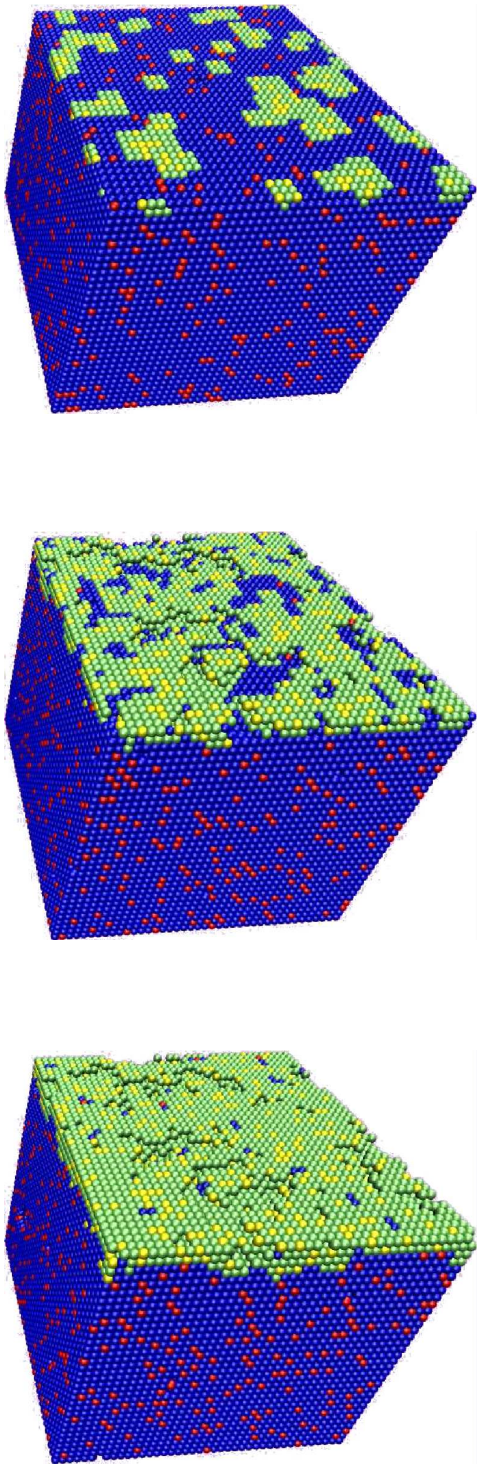

(b)

Figure 4.10: $700 \mathrm{~K}$, local threshold for passivation is $20 \%$ (a) $\mathrm{Fe}_{92} \mathrm{Cr}_{8}$ continued dissolution (b) $\mathrm{Fe}_{90} \mathrm{Cr}_{10}$ a passive film forms. Atoms colours; blue $=\mathrm{Fe}$, $\mathrm{red}=\mathrm{Cr}$, green $=$ passive $\mathrm{Fe}$, yellow $=$ passive $\mathrm{Cr}$. 
Figure 4.10 shows snapshots of the simulations in the two cases either side of the transition, $8 \%$ and $10 \% \mathrm{Cr}$ content, to illustrate the difference in behaviour. The final frame of figure 4.10(b) shows that a complete film of passive material has formed, inhibiting further dissolution. While figure 4.10(a) shows the passivated areas are too disjoint to allow a continuous film to form and hence dissolution continues.

Now we can consider the impact of the Cr-current on passive film formation. Figure 4.11 shows total dissolution curves at the same set of alloy Cr contents, $5 \%$ to $25 \%$. Again, the local threshold for surface passivation is $20 \%$. There is a clear difference in behaviour. A passive film forms at $10 \% \mathrm{Cr}$ content with the $\mathrm{Cr}$ current, but not until 15\% without.

Here, let us consider two borderline cases for passivation. Firstly, we consider the $15 \% \mathrm{Cr}$ case in figure 4.11 (b) mentioned above to be passivated. This model is designed to emulate the onset of passive film formation. It is assumed that, after the initial layer forms other mechanisms of growth not modeled here will take over. In this case, we observe the cessation of dissolution indicating the onset of passivation. After some time dissolution resumes at a fast rate. In this situation vacancies have aggregated under the passive layer, the definition of surface has been compromised, and dissolution starts occurring from the center of the block rather than the surface.

The second borderline case, is the $9 \%$ curve in figure 4.9 , which appears to be right between a passive film forming and not. The first frames of figure 4.10 show that in the $\mathrm{Fe}_{0.90} \mathrm{Cr}_{0.10}$ alloy, where a film forms, $38 \%$ of the surface is already passivated, while only $25 \%$ of the $\mathrm{Fe}_{0.92} \mathrm{Cr}_{0.08}$ is passivated at the start of the simulation. In the borderline $\mathrm{Fe}_{0.91} \mathrm{Cr}_{0.09}$ the initial surface passivation is 37\%. Perhaps if the distribution of $\mathrm{Cr}$ was different and the initial surface passivation lower, this alloy would be observed to definitively not form a passive film. So, the distribution of $\mathrm{Cr}$ at the surface could be impacting the formation of a passive film for alloys that are borderline. This effect could warrant further investigation. 


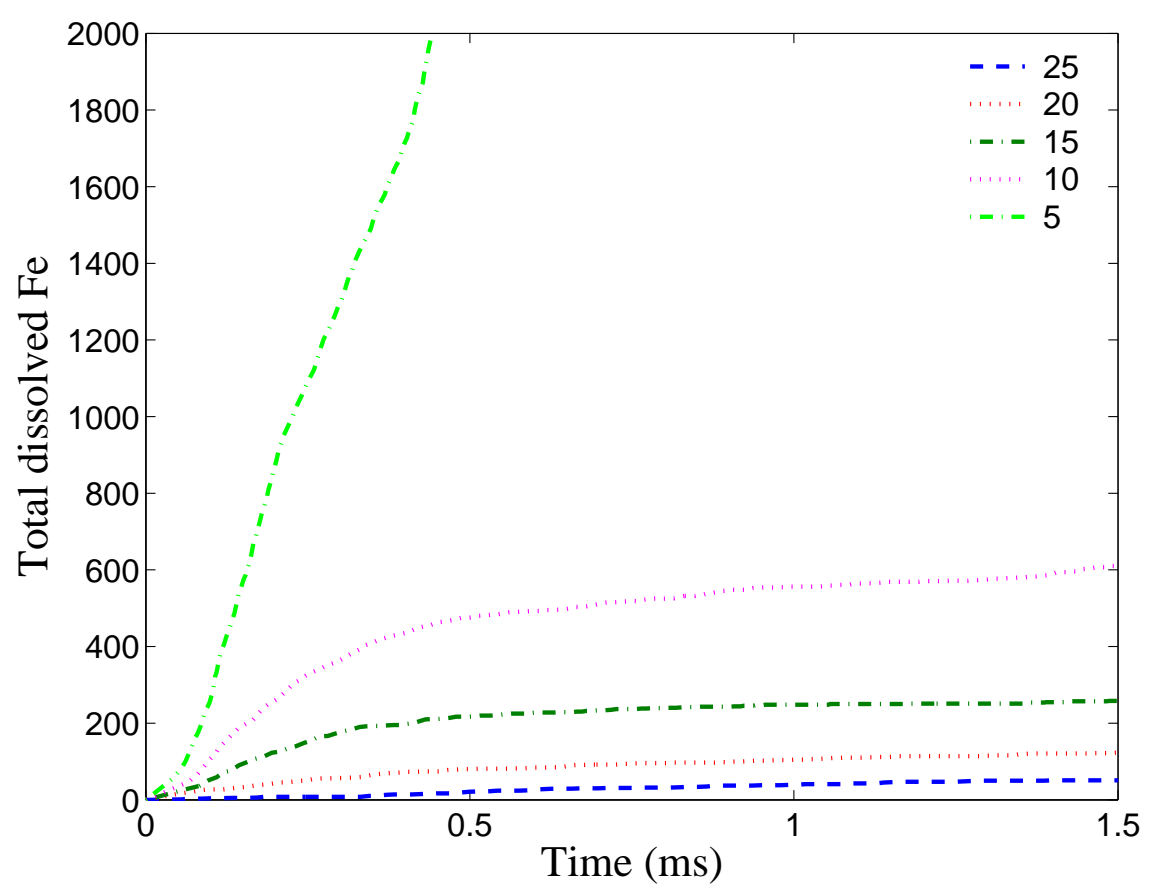

(a)

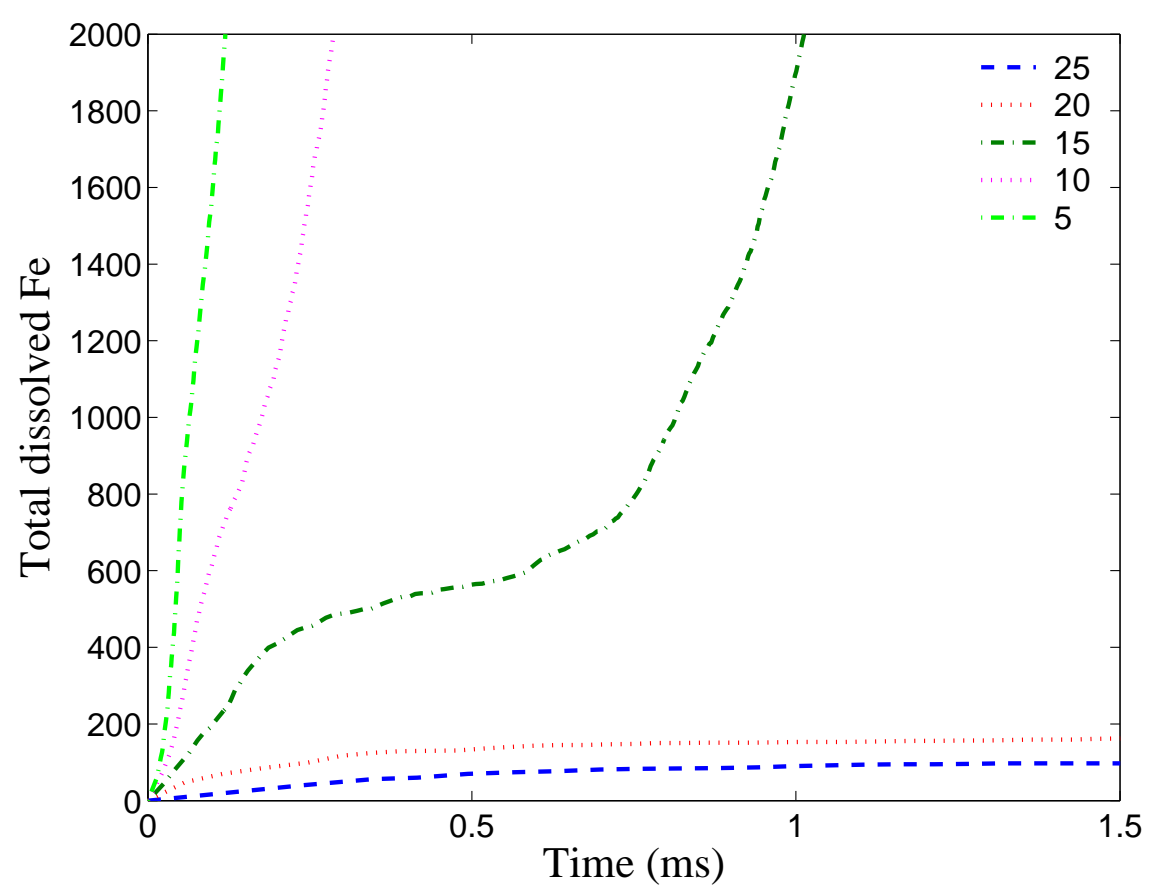

(b)

Figure 4.11: Comparison of passivation in (a) mobile-Cr regime (b) Fe-like regime, for fixed alloy mixtures from $5 \% \mathrm{Cr}$ content to $25 \% \mathrm{Cr}$ content at $700 \mathrm{~K}$. The simulation block has 34 planes parallel to the surface containing 1681 atoms each. 


\begin{tabular}{ccc}
\hline$\nu_{1}$ & Cr-Mobile & Fe-like \\
\hline \hline & & \\
$10^{-5}$ & $8 \%$ & $13 \%$ \\
$10^{-4}$ & $10 \%$ & $15 \%$ \\
$10^{-3}$ & $13 \%$ & $19 \%$ \\
& & \\
\hline
\end{tabular}

Table 4.1: Minimum bulk $\mathrm{Cr}$ content required for passive film to form when the local passivation threshold is $20 \%$.

Table 4.1 summarises the minimum $\mathrm{Cr}$ content that will result in a film forming when the local passivation threshold is set to $20 \%$. It is clear that when there is a $\mathrm{Cr}$ current the minimum bulk level of $\mathrm{Cr}$ required is around 5\% less than without. The Cr current could therefore be a significant consideration in the formation of the passive film. In particular, in experimental conditions where passivation occurs with minimal dissolution [19].

We expected that when we have a Cr current, the required content of $\mathrm{Cr}$ in the alloy to enrich the surface to a particular level decreases with decreasing dissolution rate. Figure 4.8 shows that surface enrichment occurs with less dissolution at lower dissolution rate. However, this was not the case when no $\mathrm{Cr}$ current was present. Yet, the same decreasing trend in alloy threshold is observed. This perhaps indicates some limitations in the description of passive film growth we employ.

The onset of the Cr current also fits with the sharp onset of passive film formation with increasing $\mathrm{Cr}$ content. Our analytical model predicts that there is a Cr current above $17 \% \mathrm{Cr}$ content in the alloy. First consider an alloy with $\mathrm{Cr}$ content just below this threshold in an experimental setup in which it does not passivate. Then consider an alloy with $\mathrm{Cr}$ content just above this threshold, in the same experimental setup. The latter could 
form a passive film of a higher $\mathrm{Cr}$ content.

\subsection{Summary}

This section has presented results of KMC simulations on both bulk and surface configurations. We determined that while our simplistic bond counting scheme does not reproduce $\alpha$-phase separation in the bulk, we can simulate a surface driven $\mathrm{Cr}$ current. This current enriches the surface irrespective of where vacancies originate. We found that surface enrichment occurs with less dissolution with the Cr current compared to without and found that this enrichment slows dissolution even with no modelling of passivation. We observe a threshold for passive film formation which decreases with $\mathrm{Cr}$ current, and decreasing rate of dissolution. These results are further summarised in the following section, which concludes this work. 


\section{Chapter 5}

\section{Conclusions}

Given the industrial importance of stainless steels, a thorough understanding of the factors impacting corrosion and the mechanisms of passive film formation is desirable.

Previous experimental studies have determined that the onset of passivation in $\mathrm{Fe}-\mathrm{Cr}$ alloys occurs sharply at a set $\mathrm{Cr}$ percentage, and both first principles calculations and experiments confirm the protective oxide layer which forms is enriched in $\mathrm{Cr}$ compared to the alloy itself.

We have proposed a model for the onset of passivity which relates passive film formation to an instability in the alloy. Using a Cahn-Hilliard type model and parameters fitted to ab initio data we derive the onset of a phase separating current at $17 \% \mathrm{Cr}$ content in the bulk alloy.

We took an atomistic approach to investigate the impact of a Cr current to the surface on passivation. We implemented Schulze's fast kinetic Monte Carlo algorithm with extensions to allow for vacancy assisted nearest neighbour migration, tracking a surface, dissolution and surface passivation. We modelled a surface driven $\mathrm{Cr}$ current resulting from the higher mobility of $\mathrm{Cr}$ atoms, and the larger number of events occurring at the surface.

We found that with no dissolution occurring this current still enriches the surface, irrespective of whether vacancies originate in the bulk or at 
the surface. The maximum level of enrichment reached, $40 \%$, is within the range found experimentally by Suzuki et al. [28]. Also, this current results in fewer $\mathrm{Cr}$ atoms dissolving for a set surface $\mathrm{Cr}$ enrichment compared to enrichment resulting purely from preferential Fe dissolution. What is more, the disparity increases as the dissolution rate slows. This is particularly important because experimental dissolution rates are slower than the timescales we can model computationally. This indicates our model is able to account for situations where little or no Fe dissolves, and yet a $\mathrm{Cr}$ enriched oxide film still forms, a significant limitation of previous models which rely on preferential Fe dissolution as the only mechanism for surface $\mathrm{Cr}$ enrichment.

When we modelled passive surface formation, we observed a sharp onset of passivation. We found that the threshold level of alloy $\mathrm{Cr}$ content required to form a passive surface with a particular passivation condition decreased with decreasing current. In addition, the required alloy content for passivation was consistently $\sim 5 \%$ lesser when we simulated a $\mathrm{Cr}$ current as opposed to without. This indicates that the onset of the Cr current at a particular level could result in a sharp change in behaviour. If the thermodynamically stable film for an experimental setup fell between that range, a sharp onset of passivation would occur with the onset of a current.

There are several directions in which this project could be extended.

Firstly, while $\mathrm{Cr}$ dissolution affected the rate of Fe dissolution, particularly if no $\mathrm{Cr}$ current was present, little impact was seen on the level of surface $\mathrm{Cr}$ enrichment. Given that few models allow for $\mathrm{Cr}$ dissolution, this could warrant further investigation. In our simulations up to $3 \%$ of the total dissolved material was Cr. Hamm et al. [12] observed up to $10 \%$ of the total dissolved material was $\mathrm{Cr}$ experimentally, so there is room to investigate higher rates of $\mathrm{Cr}$ dissolution.

Secondly, it is interesting that the required alloy content for passivation decreases with decreasing dissolution rate when there is no $\mathrm{Cr}$ current. 
Our dissolution simulations indicate that a set number of Fe dissolutions is required for surface $\mathrm{Cr}$ enrichment, independent of the dissolution rate. So perhaps, our mechanism for passive film formation within the KMC algorithm could require refinement.

In addition allowing 3 species in a KMC algorithm is a significant extension which could be further generalised or applied to other systems such as nanoparticles. 


\section{References}

[1] Bessone, J., Karakaya, L., Lorbeer, P., and Lorenz, W. The kinetics of iron dissolution and passivation. Electrochim. Acta. 22, 10 (1977), 1147-1154.

[2] Bhadeshia, H., Bhadeshia, H., And Honeycombe, R. Steels: microstructure and properties. Butterworth-Heinemann, 2006.

[3] Blue, J., Beichl, I., AND Sullivan, F. Faster Monte Carlo simulations. Phys. Rev. E 51, 2 (Feb 1995), R867-R868.

[4] Bonny, G., Terentyev, D., Malerba, L., and Van Neck, D. Early stages of $\alpha-\alpha^{\prime}$ phase separation in $\mathrm{Fe}-\mathrm{Cr}$ alloys: An atomistic study. Phys. Rev. B 79, 10 (Mar 2009), 104207.

[5] BortZ, A., Kalos, M., AND Lebowitz, J. A new algorithm for Monte Carlo simulation of ising spin systems. J. Comp. Phys. 17, 1 (1975), 10-18.

[6] CAhN, J., AND Hilliard, J. Free energy of a nonuniform system. i. interfacial free energy. J. Chem. Phys. 28 (1958), 258.

[7] Caro, A., Crowson, D., And Caro, M. Classical many-body potential for concentrated alloys and the inversion of order in ironchromium alloys. Phys. Rev. Lett. 95, 7 (2005), 75702.

[8] Chen, D., GaO, F., Hu, W. Y., Hu, S. Y., Terentyev, D., Sun, X., Heinisch, H. L., Henager, C. H., And Khaleel, M. A. Migration 
of Cr-vacancy clusters and interstitial $\mathrm{Cr}$ in $\alpha-\mathrm{Fe}$ using the dimer method. Phys. Rev. B 81, 6 (Feb 2010), 064101.

[9] Choudhury, S., Barnard, L., Tucker, J., Allen, T., Wirth, B., ASTA, M., AND MORGAN, D. Ab-initio based modeling of diffusion in dilute bcc Fe-Ni and $\mathrm{Fe}-\mathrm{Cr}$ alloys and implications for radiation induced segregation. J. Nucl. Mater. 411 (2011), 1-14.

[10] Clarke, S., AND Vvedensky, D. Growth kinetics and step density in reflection high-energy electron diffraction during molecular-beam epitaxy. J. Appl. Phys. 63, 7 (1988), 2272-2283.

[11] Davenport, A., Oblonsky, L., Ryan, M., And Toney, M. The structure of the passive film that forms on iron in aqueous environments. J. Electrochem. Soc. 147 (2000), 2162.

[12] Hamm, D., Ogle, K., Olsson, C., Weber, S., and Landolt, D. Passivation of Fe-Cr alloys studied with icp-aes and eqcm. Corr. Sci. 44, 7 (2002), 1443-1456.

[13] INGHAM, B., HENDY, S., LAYCOCK, N., AND RYAN, M. Estimates of thermodynamic stability of iron-chromium spinels in aqueous solution based on first-principles calculations. Electrochem. Solid State Lett. 10 (2007), C57.

[14] Kittel, C., And Kroemer, H. Thermal Physics, 2nd edition ed. W. H. Freeman, 1980.

[15] Legrand, M., Diawara, B., Legendre, J., And Marcus, P. Three-dimensional modelling of selective dissolution and passivation of iron-chromium alloys. Corr. Sci. 44, 4 (2002), 773-790.

[16] MCCAFFERTY, E. Graph theory and the passivity of binary alloys. Corr. Sci. 42, 11 (2000), 1993-2011. 
[17] MCCAFFERTY, E. Graph theory and the passivity of binary alloys. J. Electrochem. Soc. 151 (2004), B82.

[18] OBlOnsky, L., RYAN, M., AND IsAaCs, H. In situ determination of the composition of surface films formed on Fe-Cr alloys. J. Electrochem. Soc. 145 (1998), 1922.

[19] OBLONSKy, L., RYAN, M., AND IsAaCS, H. In situ determination of the composition of surface films formed on $\mathrm{Fe}-\mathrm{Cr}$ alloys. J. Electrochem. Soc. 145 (1998), 1922.

[20] OBLONSKY, L., RYAN, M., AND ISAACS, H. In situ xanes study of the formation and reduction of the passive film formed on Fe in acetate solution. Corr. Sci. 42, 2 (2000), 229-241.

[21] Olsson, C., AND LANDOlt, D. Passive films on stainless steelschemistry, structure and growth. Electrochim. Acta. 48, 9 (2003), 10931104.

[22] Olsson, P., Wallenius, J., Domain, C., Nordlund, K., AND MALERBA, L. Two-band modeling of $\alpha$-prime phase formation in Fe-Cr. Phys. Rev. B 72, 21 (2005), 214119.

[23] Qian, S., NeWman, R., CotTis, R., AND SieradzKI, K. Validation of a percolation model for passivation of Fe-Cr alloys: twodimensional computer simulations. J. Electrochem. Soc 137, 2 (1990), 435.

[24] Ryan, M., Newman, R., And Thompson, G. Atomically resolved stm of oxide film structures on Fe-Cr alloys during passivation in sulfuric acid solution. J. Electrochem. Soc. 141 (1994), L164.

[25] SAunders, N., And Miodownik, A. CALPHAD: A Comprehensive Guide. Pergamon Materials Series, 1998. 
[26] Schulze, T. Kinetic Monte Carlo simulations with minimal searching. Phys. Rev. E 65, 3 (2002), 036704.

[27] SERADZKI, K., AND NEWMAN, R. A percolation model for passivation in stainless steels. J. Electrochem. Soc 134 (1987), 1635.

[28] Suzuki, S., Kosaka, T., InOue, H., Isshiki, M., AND WAseda, Y. Effect of the surface segregation of chromium on oxidation of highpurity Fe-Cr alloys at room temperature. Appl. Surf. Sci. 103, 4 (1996), 495-502.

[29] TONey, M., DAVEnPort, A., Oblonsky, L., RYAn, M., AND Vitus, C. Atomic structure of the passive oxide film formed on iron. Phys. Rev. Lett. 79, 21 (1997), 4282-4285.

[30] Voter, A. Introduction to the kinetic Monte Carlo method. Rad. Effects in Solids (2007), 1-23.

[31] Wallenius, J., Abrikosov, I., Chakarova, R., Lagerstedt, C., Malerba, L., Olsson, P., Pontikis, V., SAndberG, N., AND TerENTYEV, D. Development of an eam potential for simulation of radiation damage in Fe-Cr alloys. J. Nucl. Mater. 329 (2004), 1175-1179.

[32] Wallenius, J., Olsson, P., Lagerstedt, C., SandberG, N., Chakarova, R., AND PONTIKIS, V. Modeling of chromium precipitation in Fe-Cr alloys. Phys. Rev. B 69, 9 (2004), 094103. 